\title{
Oblique Nuclear Quadrupole Interaction in Self-Assembled Structures Based on Semiconductor Quantum Dots
}

\author{
Y.Q. Huang ${ }^{*},{ }^{*}$ Y. Puttisong, S. Filippov, I.A. Buyanova $₫$, and W.M. Chen $\oplus^{\dagger}$ \\ Department of Physics, Chemistry and Biology, Linköping University, S-581 83 Linköping, Sweden
}

(Received 11 October 2019; revised 27 August 2020; accepted 28 August 2020; published 13 October 2020)

\begin{abstract}
Dynamic nuclear polarization (DNP) is well recognized as being important in spintronics and quantuminformation processing. DNP gives rise to high nuclear spin polarization that not only can prolong electron-spin lifetime by generating an Overhauser field (OHF), but also has fertilized the idea to explore nuclear spin qubits. In strained quantum-dot structures (QDSs), a nuclear spin is coupled to a strain field via its quadrupole moment. It has been shown that such nuclear quadrupole interaction (NQI) can be used to achieve appreciable DNP and hence electron-spin polarization. Here, we uncover magneto-optical anomalies from a series of laterally arranged (In, Ga)As QDSs that arise from the NQI and DNP in these nanostructures. We find that the principal axis of NQI in symmetry-lowered QDSs significantly deviates from the growth direction, resulting in tilting of OHF with an angle exceeding $37^{\circ}$. The resulting transverse component of OHF is probed with respect to the crystallographic orientations and its influence on the DNP and ensemble spin dephasing is analyzed. We show that a high-symmetry electronic confinement potential for excitons does not guarantee a high-symmetry NQI for nuclei within the same nano-object, thereby calling for correlated optimization in the symmetry of the electronic confinement potential and that of the nuclear spin bath. Our results underline the role of oblique NQI in electron-spin decoherence and depolarization, which has so far largely been overlooked. This work thus sheds light on design rules for engineering the electronic and spin landscape of QDSs for better performance of DNP desirable for applications in spintronics and quantum computation.
\end{abstract}

DOI: 10.1103/PhysRevApplied.14.044019

\section{INTRODUCTION}

Dynamic nuclear polarization (DNP) in semiconductor quantum-dot structures (QDSs) has been a subject of intense studies over recent decades [1-5]. This is not only because it represents a promising means to suppress electron-spin decoherence from random nuclear spin fluctuations in nuclear-spin-rich materials, but also it is at the heart of various efforts to implement nuclear spin qubits for quantum-information processing (QIP) and spintronic applications [6-9]. DNP is built up through the hyperfine interaction such that angular momentum is transferred from an electron spin to a nuclear spin. One issue of the DNP process is the presence of nuclear spin-spin interactions, which generate a local field $B_{L}$ that rapidly depolarizes the nuclear spins at zero external magnetic field. It was recognized that the key to suppress the nuclear spin

\footnotetext{
*yuqing.huang@liu.se

†Weimin.chen@liu.se

Published by the American Physical Society under the terms of the Creative Commons Attribution 4.0 International license. Further distribution of this work must maintain attribution to the author(s) and the published article's title, journal citation, and DOI. Funded by Bibsam.
}

loss and to recover DNP in strained QDSs stems from the nuclear quadrupole interaction (NQI) [1]. By assuming a strain axis along the growth direction, it has been shown that NQI decouples the nuclear spins from fluctuating $B_{L}$ and thus stabilizes the electron and nuclear spin orientation. This is confirmed by the observation of a broadened Hanle curve and the effect of NQI has widely been acquired to explain the observations of suppressed nuclear spin diffusion [10], anomalous Hanle effect [11], electronspin decoherence dynamics in strained QDSs [12-15]. Recently, by applying the technique of optically detected nuclear magnetic resonance (ODNMR), the effect of NQI is directly demonstrated by correlating an ultralong nuclear spin-echo coherence time with the presence of a finite quadrupole shift in the nuclear-spin-rich and strained QDs [16-18].

Despite various efforts being made regarding the NQI effect in QDs, little is understood about the anisotropy of NQI and its effect on DNP. This is mainly due to the oversimplified NQI model that assumes a dominant uniaxial strain field with its principal axis along the growth direction. In reality, however, effects like an inhomogeneous strain and charge distribution or atomistic randomness within a QD or charge fluctuations between neighboring QDs can significantly alter the local electric field 
gradient at different atomic sites such that the principal axes of NQI for individual nuclei are not necessarily oriented along the growth direction [19,20]. Such a spread of nuclear spin interactions is believed to be responsible for the observation of the broadened ODNMR signal and an intermediate stage of electron-nuclear spin relaxation [18]. However, since most early studies only involve a single QD with symmetry of $C_{2 v}$ or higher such that the anisotropy of NQI is averaged out, a direct probe of its effect on DNP is not accessible so far. This is despite the fact that QD arrays, which are at the core of many ideas for solid-state implementations of scalable QIP using electron spins, are typically accompanied by a low-symmetry strain field due to the presence of surrounding interacting QDs where a better understanding and control of the anisotropic NQI and its effect on electron spins are required.

In this work, we examine the anisotropic effect of NQI in a series of laterally arranged (In, Ga)As QDSs that have a symmetry lower than $C_{2 v}$ that is typical for isolated QD. Unlike the symmetric QDSs, the average principal axis of NQI in these nanostructures is found to significantly deviate from the [001] growth direction, resulting in an appreciable transverse component of the Overhauser field (OHF). The in-plane angular orientation of the transverse OHF is measured to uncover the preferable orientation of the NQI axis in different QDSs, and depolarization of electron spins in the transverse OHF is illustrated. Our results call for special attention to be given to the role of anisotropic NQI in asymmetric QDSs and its detrimental effect on spin coherence.

\section{EXPERIMENTAL DETAILS}

The studied structures are a set of (In, Ga)As QDSs grown by molecular beam epitaxy on a GaAs (001) substrate. The growth conditions that lead to the formation of different QDSs are found in Note 1 within the Supplemental Material [21]. For optical orientation spectroscopy, the sample is placed in a liquid-helium continuous-flow cryostat $(T=6 \mathrm{~K})$ and is optically pumped by circularly polarized light from a cw Ti-sapphire laser propagating along the surface normal direction. The excitation is nonresonant with a photon energy $(1.59 \mathrm{eV})$ above the GaAs bandgap energy and the helicity of excitation is fixed at either $\sigma^{+}$or $\sigma^{-}$to allow build up of DNP. The excitation photon energy is chosen to maximize the DNP such that the subtle effects due to oblique NQI can be identified. In control experiments, DNP is avoided by modulating the excitation polarization between $\sigma^{+}$and $\sigma^{-}$at a frequency of $50 \mathrm{kHz}$, which is too fast for DNP to build up. The measurement configurations with and without the build up of DNP is schematically illustrated in more details in Fig. S1 within the Supplemental Material [21]. PL from the QDSs is detected in a backscattering geometry and circular polarization degree of the emission, $P_{\mathrm{PL}}=\left(I^{+}-I^{-}\right)$
$/\left(I^{+}+I^{-}\right)$, is derived within the PL peak [21]. The magneto-optical characterizations are done only for the QDS ensembles, which allows angular-dependent studies by rotating the sample with respect to the applied magnetic field. They are carried out with the aid of a conventional electromagnet, where the magnetic field is controlled with a feedback loop and calibrated independently with a gauss meter. The stray field is limited to be less than $7 \mathrm{G}$ in the system. The external magnetic field $\boldsymbol{B}$ is applied along (or perpendicular to) the light propagating direction in the Faraday (or Voigt) geometry. The scanning speed of the field is fixed at $33 \mathrm{G} / \mathrm{min}$ for the $2000-\mathrm{G}$ scanning range and $0.66 \mathrm{G} / \mathrm{min}$ for the $40-\mathrm{G}$ scanning range. At both scanning speeds, no distortion of the spectra is observed.

\section{RESULTS AND DISCUSSION}

\section{A. Quantum-dot structures with low symmetries}

The atomic-force-microscope (AFM) images of the QDSs are shown in Figs. 1(a)-1(c), including quantum clusters (QCs), quantum rings (QRs), and double QDs (DQDs). All the QDSs are formed by applying partial capping and postgrowth of single-QD samples under different conditions [22]. As shown by the AFM images, the QC is made of few, typically six, QDs, laterally arranged to a ringlike structure, whereas the $\mathrm{QR}$ has a real ring shape. The DQD consists of two QDs laterally arranged along the [110] crystallographic axis. Despite the seemingly symmetric lateral arrangement of the QDSs, owing to growth mechanism, the actual QDSs usually deviate from the symmetric structures. For instance, as found by Offermans et al., owing to a difference in indium migration speed along two different $\langle 110\rangle$ axes, the QR has higher ridges along the [110] direction [23]. This is also seen in the three-dimensional (3D) reconstruction of the AFM image of individual QDSs shown in Figs. 1(d)-1(f). Due to a size variation of the constituent QDs in a QC or DQD, or a height variation along the $\mathrm{QR}$ ridge, the carriers confined in the QDS ground states are then localized to only part of the nanostructure [24]. This can be either the larger-size constituent QD within a QC or DQD [QD "A" in Fig. 1(d) or "F" in Fig. 1(f)], or a higher ridge of a QR [ridge "D" in Fig. 1(e)]. The presence of neighboring QDs or ridges [QD "C" and "B" in Fig. 1(d), ridge "E" in Fig. 1(e), and QD "G" in Fig. 1(f)] perturb the strain and alloy distribution within the carrier localization volume, reducing the symmetry to $C_{s}$ or lower. The asymmetric strain and alloy condition of the QDSs, if not completely random, have finite effects on the orientation of electron and nuclear spin in those nanostructures, which is demonstrated below.

\section{B. Effect of a transverse magnetic field on dynamic nuclear polarization}

All the QDS samples are found to be slightly $p$-type doped, evident from the observation of the PL emission 
(a)

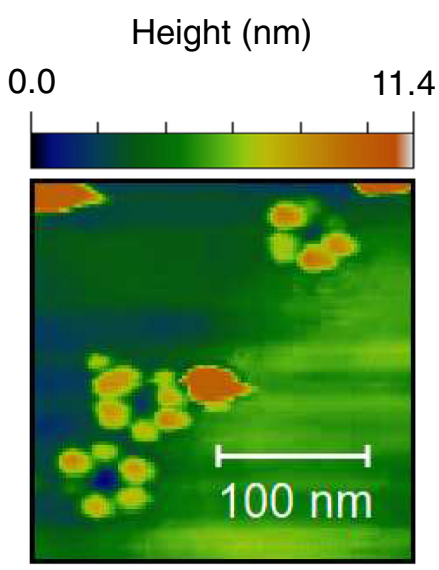

(d)

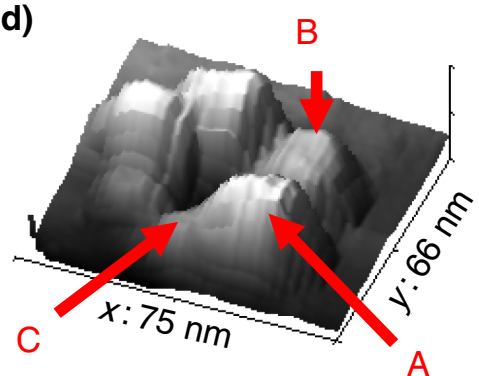

(b)

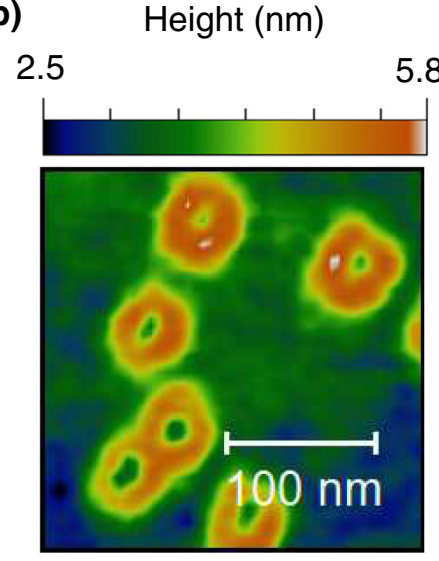

(e)

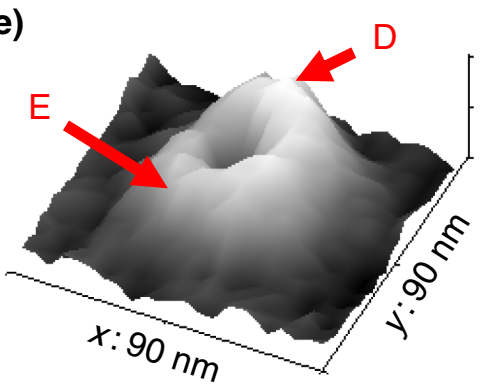

(c) Height (nm)

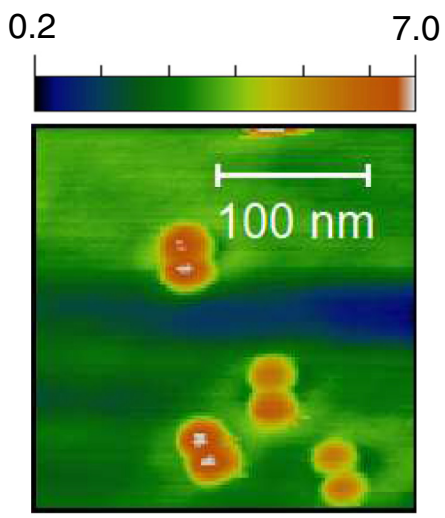

(f)

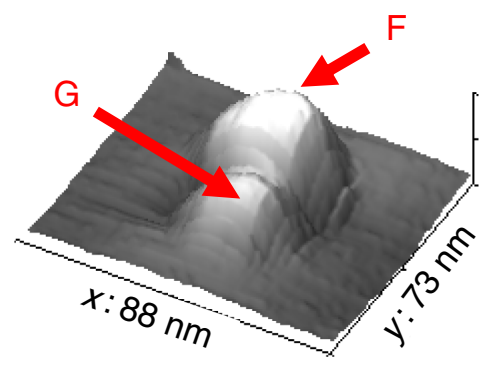

FIG. 1. Quantum-dot structures with lowered symmetry. (a)-(c) Atomic force microscopic images of the studied QD clusters (QCs), quantum rings (QRs), double QDs (DQDs), respectively. (d)-(f) 3D reconstruction of the AFM close-up images of individual QC, $\mathrm{QR}$, and DQD. The features leading to the low symmetries of the nanostructures, which are discussed in the main text, are labeled by $\mathrm{A}-\mathrm{G}$.

related to the $C_{\mathrm{As}}$ acceptor (see Fig. S2 within the Supplemental Material [21]) [25]. As a result of such residual doping, the PL spectra from the studied QDSs are mainly contributed from the positively charged trion $X^{+}$. In optical orientation experiments [26], the radiative recombination of $X^{+}$with partially preserved spin polarization leads to the characteristic copolarized PL emission that is observed in all of the studied QDS samples (see Fig. S3 within the Supplemental Material [21]). The predominant $X^{+}$emission in our samples provides a perfect probe to study the electron-nuclear spin interaction. Since the spins of the two holes within the $X^{+}$complex are paired off, the selection rules of the radiative recombination is then determined solely by the electron-spin polarization. The circular polarization degree of the emission, $P_{\mathrm{PL}}=$ $\left(I^{+}-I^{-}\right) /\left(I^{+}+I^{-}\right)$, thus provides a direct measure of the spin polarization of the electron in the $X^{+}$complex. Here, $I^{+}\left(I^{-}\right)$denotes the intensity of the $\sigma^{+}\left(\sigma^{-}\right)$PL component. Moreover, as both the electron-hole exchange interaction and the Fermi contact hyperfine interaction of the holes vanish in $=$, the Hamiltonian of the system can be simplified to the interaction only associated with the electron and nuclear spins. Apart from the dominant $X^{+}$ component, the emission of the neutral exciton $X^{0}$ is also present in our samples. $X^{0}$ is featured by a nonvanished anisotropic exchange interaction (AEI) that gives rise to a fine structure consisting of two linearly and cross-polarized lines resolved in a micro-PL spectrum [27]. As a result, $P_{\mathrm{PL}}$ of $X^{0}$ is generally weak in zero and weak magnetic fields, which reduces the overall $P_{\mathrm{PL}}$ from the studied QDSs. However, we should point out that $X^{0}$, usually reported to have a spin lifetime less than $100 \mathrm{ps}$, should give rise to a broad background in the Hanle spectra [28] that has a negligible effect on the Hanle effect in the low-field regime (with a transverse magnetic field $\left|\mathbf{B}_{\perp}\right|<100 \mathrm{G}$ ) focused in this work. In the Faraday geometry, the longitudinal magnetic field $\mathbf{B}_{\|}$recovers the spin states of $X^{0}$ and produces a slow-varying monotonic increase of $P_{\mathrm{PL}}$ as a function of $\left|\mathbf{B}_{\|}\right|$[29], which neither affects the results to be presented below.

Figure 2(a) shows representative Hanle curves obtained from the QR sample in an external transverse magnetic field $\mathbf{B}_{\perp}$. The measurements are carried out in the presence of DNP under continuous illumination of $\sigma^{+}$-polarized light propagating along the surface normal direction. The applied transverse magnetic field is rotated within the sample surface plane and the rotation angle $\phi$ is defined with respect to the [110] crystal axis. The Hanle effect characterizes spin depolarization induced by the spin precession in the transverse magnetic field, which normally give rise to a 

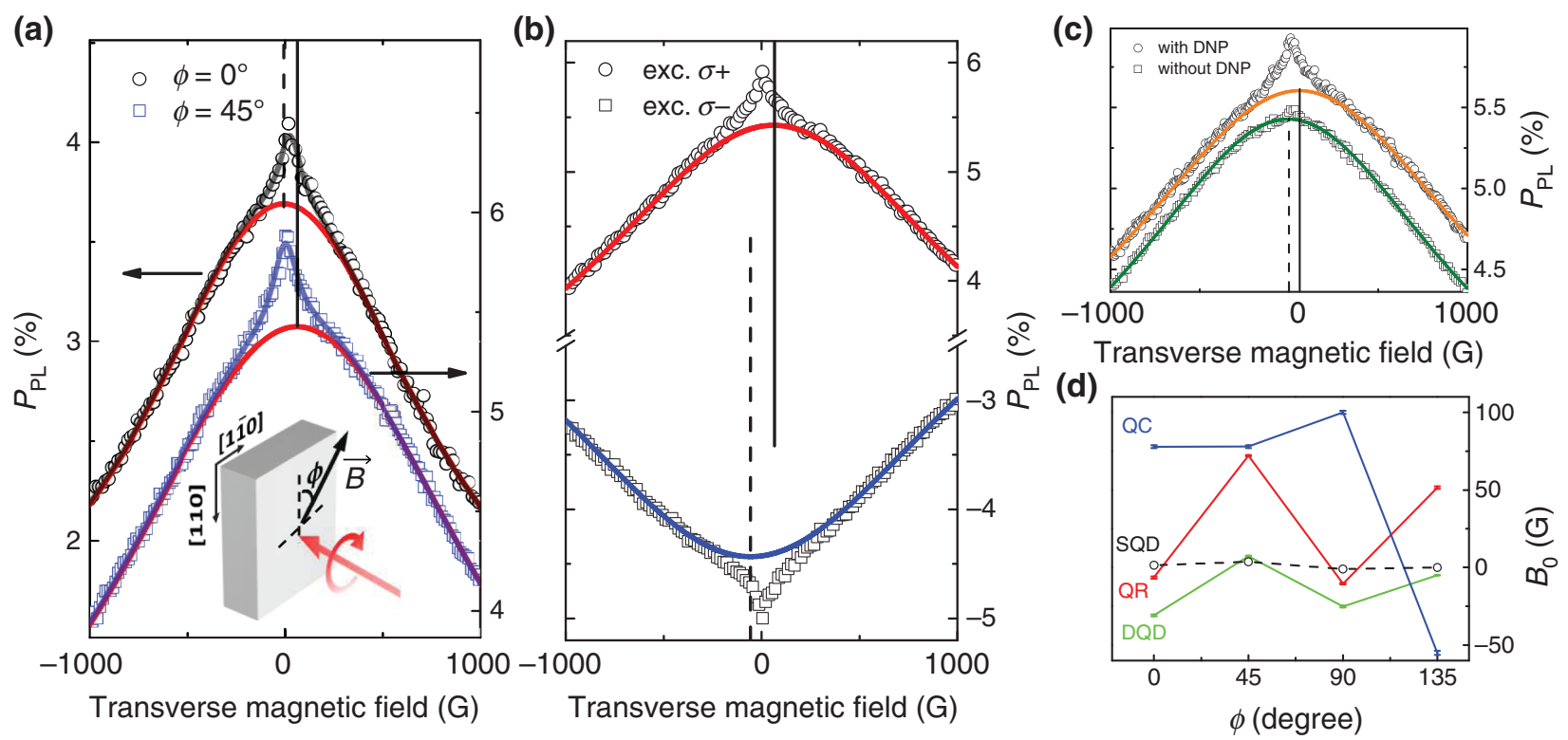

FIG. 2. Effect of a transverse magnetic field on DNP. (a) Hanle curves with different orientations of the external transverse magnetic field, obtained at $6 \mathrm{~K}$ from the QR sample under optical orientation in the Voigt geometry. $\phi$ is the in-plane rotation angle of the transverse field as illustrated in the inset. $\sigma^{+}$polarized light is used for optical orientation and the excitation power is kept at $100 \mathrm{~mW}$. (b) Hanle curves with $\phi=45^{\circ}$, obtained from QR with opposite excitation helicities. (c) Hanle curves with $\phi=45^{\circ}$, obtained from QR with and without the DNP effect. In (a)-(c), the symbols are the experimental data and the solid lines are the fitting curves of the broad Hanle component, whereas the solid and dashed vertical lines mark the offset field $B_{0}$. (d) Angular dependence of $B_{0}$ measured from the QC, QR, and DQD samples. $B_{0}$ from the SQD sample is shown by the dashed line as a reference.

Lorentzian-like field dependence of $P_{\mathrm{PL}}$ that is symmetric around zero field with the half-width $B_{1 / 2}$ determined by the spin lifetime [26]. However, the Hanle curves shown in Fig. 2(a) clearly deviate from the standard form with its lineshape seemingly depending on the orientation of the applied transverse field. For easy reference, we tentatively decompose each Hanle curve into two Lorentzian components with two distinctly different half-widths. The narrow component has a half-width of $B_{1 / 2}=64 \mathrm{G}(75 \mathrm{G})$ for $\phi=0^{\circ}\left(45^{\circ}\right)$, whereas the broad one (the red solid curve) has a much larger $B_{1 / 2}=895 \mathrm{G} \quad(1181 \mathrm{G})$. While the center of the narrow component always lies in the vicinity of zero field, the center of the broad component is shifted by an appreciable amount $B_{0}$ from zero field. Moreover, we find that $B_{0}$ is sensitive to the transverse-field orientation. As the field is rotated from $\phi=0^{\circ}$ to $45^{\circ}, B_{0}$ changes from $-6.5 \mathrm{G}$ to $72 \mathrm{G}$. as indicated by the dashed and solid vertical line in Fig. 2(a), respectively. We should note that the observation of the asymmetric Hanle curve and the nonvanishing $B_{0}$ is not a special case that is only limited to the studied QR sample, but it is a general trend that has been observed in all the measured QDSs (see Figs. S4-S6 within the Supplemental Material [21]).

First of all, the observed magneto-optical anomalies in the Voigt geometry are a direct consequence of the excitation helicity. In Fig. 2(b), we show that, for a fixed $\phi=45^{\circ}, B_{0}$ changes sign when the excitation helicity is reversed. Secondly, the effect must be related to DNP and its associated electron-nuclear spin interaction. This is because, in the absence of DNP, the measured Hanle curves recover to their normal shape as shown in Fig. 2(c), when the narrow component and $B_{0}$ of the broad component vanish. In a single QDS occupied by $X^{+}$, the hyperfine interaction couples the optically oriented electron spin with nuclear spins. This mutually generates an effective magnetic field upon each other. Namely, nuclear spin polarization creates an Overhauser field $\mathbf{B}_{N}$ acting on the electron and the polarized electron spin casts a Knight field $\mathbf{B}_{\text {Knight }}$ on the nuclear spins. In the vicinity of $B_{\perp}=0$, DNP is accomplished by a finite $\mathbf{B}_{\text {Knight }}$ and also by NQI in the case of a strained QDS [1,30]. Both $\mathbf{B}_{\text {Knight }}$ and NQI suppress the nuclear spin flip-flop process and generate an appreciable $\mathbf{B}_{N}$ that in turn stabilizes the electron-spin polarization along the surface normal direction. Due to a weak strength of $\mathbf{B}_{\text {Knight }}$, even a weak transverse external magnetic field overcomes the Knight field and reduces the nuclear spin polarization, thereby leading to electron-spin depolarization [2,31]. This gives rise to a rapid drop of $P_{\mathrm{PL}}$ in the lowfield range, which is the origin of the narrow component centered around $B_{\perp}=0$. Beyond this narrow field range, field dependence of DNP becomes weaker. As a result, the broad component of the Hanle curve can be approximated as the electron-spin precession in a stationary total field of $\mathbf{B}_{\perp}+\mathbf{B}_{N}$. The center of the broad component is thus 
at the compensation point of $B_{0}+B_{N}^{T}(\phi)=0$. Here, $B_{N}^{T}$ is the transverse component of $\mathbf{B}_{N}$ along the direction of the applied external field. By rotating the transverse-field direction in the sample surface plane, we obtain the angular dependence of $B_{0}=-B_{N}^{T}(\phi)$ from different QDS samples and the results are plotted in Fig. 2(d).

We should note that the physical origin of the observed Hanle curves are markedly different from the W-shaped Hanle curves reported in strain-free QDs, which consist of a middle sharp component and two broad side wings [2]. In the latter case, nuclear magnetization is dominated by the effect of $\mathbf{B}_{\text {Knight, }}$, which reverses its transverse-component direction when the external magnetic field is reversed. This gives rise to the appearance of two compensation points located symmetrically in positive and negative field. In our case, on the other hand, only one compensation point is identified and the as-determined $B_{N}^{T}(\phi)$ is expected to originate from a preferable direction of the nuclear spins tilting away from the surface-normal direction. Moreover, since the measured $P_{\mathrm{PL}}$ is rather weak in our sample, the magnitude of $\mathbf{B}_{\text {Knight }}$ is only a few gauss as indicated from the longitudinal-field results shown in Figs. 3(b) and 3(c). This value is about 2 orders of magnitude smaller than that reported in Ref. [2], which suggests a much weaker effect of $\mathbf{B}_{\text {Knight }}$ in our system and excludes the Knight field effect as the cause of the observed anomaly.

\section{Effect of a longitudinal magnetic field on dynamic nuclear polarization}

Next, we maintain the measurement conditions but switching to the Faraday geometry, where the external magnetic field is applied along the direction of the optical orientation axis, i.e., the surface-normal direction. In Fig. 3(a), we show the measured $P_{\mathrm{PL}}$ as a function of applied longitudinal field $\mathbf{B}_{\|}$under the condition without or with the effect of DNP. Without DNP, $P_{\mathrm{PL}}$ monotonously increases as we ramp up the magnetic field strength, which is typically observed in QDs due to the suppression of nuclear-field fluctuations or AEI $[27,32,33]$ by $\mathbf{B}_{\|}$. In this case, the minimum $P_{\mathrm{PL}}$ occurs at $B_{\|}=0$. With DNP, on the other hand, such dephasing and/or relaxation processes are readily suppressed partly by DNP and, as a consequence, higher $P_{\mathrm{PL}}$ is observed. In addition, the field position of the minimum $P_{\mathrm{PL}}$ now shifts to a finite-field value $B_{\|}=-B_{N}^{L}$ where the longitudinal component of the Overhauser field $\left(B_{N}^{L}\right)$ is fully compensated by the applied $\mathbf{B}_{\|}$. In the example case of the QR sample, $B_{N}^{L}$ is estimated to be around $335 \mathrm{G}$. If taking $B_{N}^{T}\left(45^{\circ}\right)=72 \mathrm{G}$ deduced from the Hanle result given in Fig. 2(d), we can estimate the tilting angle $\theta$ of the Overhauser field from the surface-normal direction through a simple relation $\tan \theta=B_{N}^{T}(\phi) / B_{N}^{L}$, which is around $12^{\circ}$. This value rises up to $37^{\circ}$ in the QC sample. Considering that, in the Faraday (Voigt) geometry, the
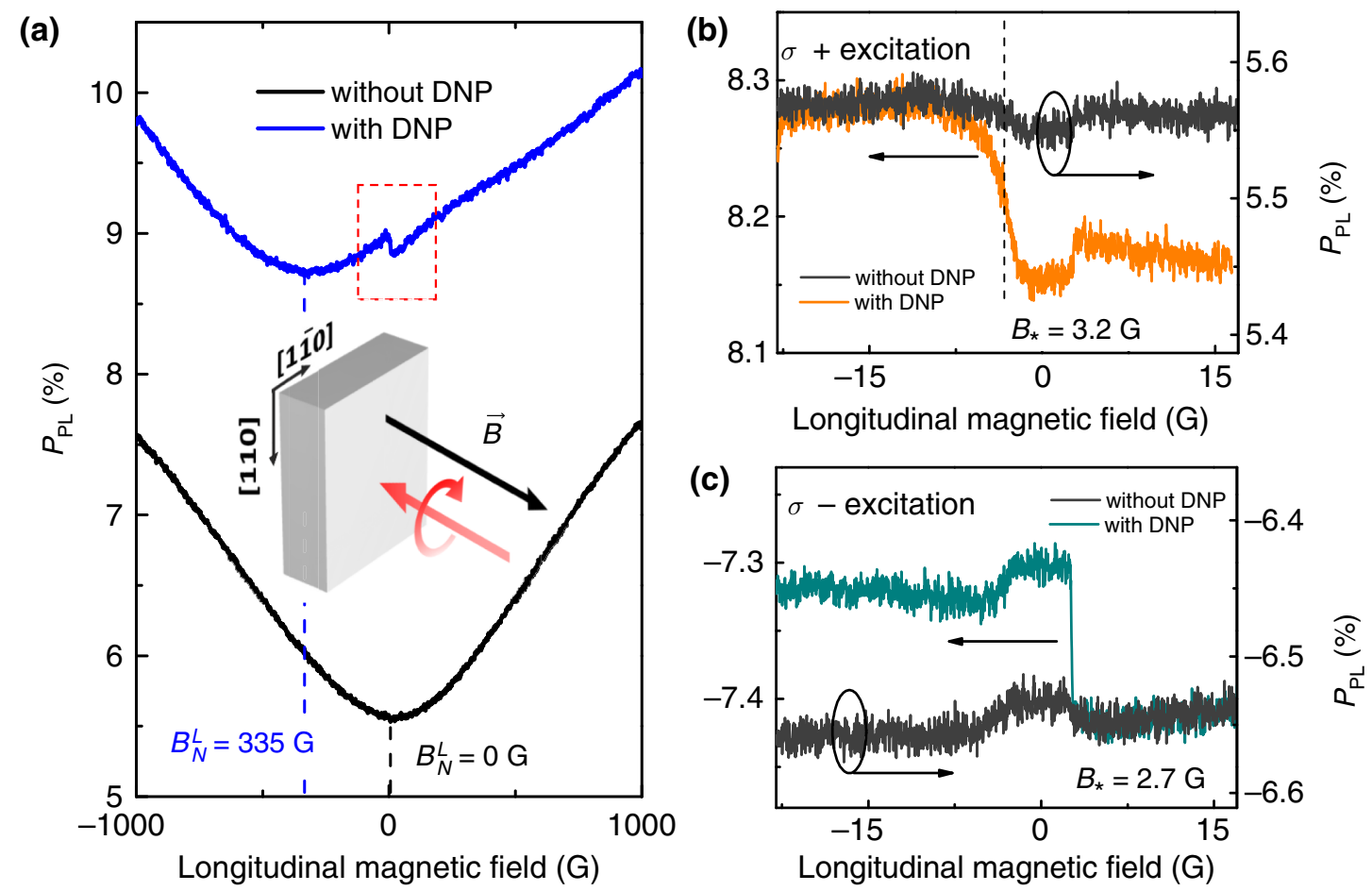

FIG. 3. Effect of a longitudinal magnetic field on DNP. (a) $P_{\mathrm{PL}}$ as a function of the external longitudinal magnetic field with and without DNP, obtained at $6 \mathrm{~K}$ from the QR sample under optical orientation with $\sigma^{+}$polarized light in the Faraday geometry. (b), (c) Close-up spectra around zero field under $\sigma^{+}$and $\sigma^{-}$excitation, respectively. 
DNP is enhanced (suppressed) due to finite longitudinal (transverse) magnetic field, the estimated $B_{N}^{L}\left(B_{N}^{T}\right)$ is actually higher (lower) than that in the absence of the external magnetic field, meaning that the real $\theta$ without applying any magnetic field would be even larger. We should note that such a large tilting angle is not possible to induce by any unintentional misalignment of the sample orientation or the applied magnetic field [34]. This entails that the tilting of the Overhauser field must originate from some actual physical processes inside the QDS.

Another unexpected feature of the results in the longitudinal field occurs at the low-field regime as highlighted by the dashed rectangle in Fig. 3(a). As mentioned above, a finite effective longitudinal field is necessary for the nuclear spins to suppress dipole-dipole depolarization and to build up DNP. This condition is violated at the point where the longitudinal component of $\mathbf{B}_{\text {Knight }}$ is compensated by the applied $\mathbf{B}_{\|}$, which leads to rapid relaxation of nuclear spins as well as a drastic decrease of $P_{\mathrm{PL}}$. Indeed, it has been shown for QD ensembles that a narrow "dip" of $P_{\mathrm{PL}}$ is created at finite $B_{\|}$and the position of the polarization "dip" represents well the magnitude of $\mathbf{B}_{\text {Knight }}[30,35]$. In the upper curve of Fig. 3(a), at the compensation point of $\mathbf{B}_{\text {Knight }}, P_{\mathrm{PL}}$ exhibits a steplike feature that is rather different from that commonly reported in the literature [35]. To examine this feature in more detail, we carry out measurements within a narrow-field range both without and with DNP. In the latter case, different helicities of the pumping light $\left(\sigma^{+}\right.$and $\left.\sigma^{-}\right)$are also tested as shown in Figs. 3(b) and 3(c) for the QR sample as an example. From these results, we confirm, first of all, that the steplike feature is only visible when DNP is present. Secondly, the shape and position of the feature depend on the excitation helicity. For instance, under $\sigma^{+}$excitation, the discontinuity of $P_{\mathrm{PL}}$ occurs at $B_{*}=-3.2 \mathrm{G}$ and the PL polarization degree $\left|P_{\mathrm{PL}}\right|$ is lower for $B_{\|}>B_{*}$. After switching the excitation helicity, the discontinuity changes to $B_{*}=2.7 \mathrm{G}$ and the "step" is also reversed such that $\left|P_{\mathrm{PL}}\right|$ is lower for $B_{\|}<B_{*}$. Similar results are also obtained from the DQD and QC samples (see Fig. S7 within the Supplemental Material [21]). The overall "step" feature follows the switching of the excitation helicity that reverses the polarization of the orientated electron spin and nuclear spins such that both Knight field and Overhauser field are inverted. This naturally leads to the sign change of $B_{*}$ and the Overhauser shift $B_{N}^{L}$ as observed here. Since the strength of $B_{*}$ in Fig. 3 is on the order of a typical stray field in the system, the accurate determination of $B_{*}$ is challenging. This might explain the slight asymmetry between $\sigma^{+}$ and $\sigma^{-}$excitation seen between Figs. 3(b) and 3(c). However, we note the "step" feature, which also depends on excitation helicity, cannot be explained by a stray field from any unintentionally magnetization of nearby objects. To further ensure the stray-field effect is negligible, we repeat the measurement by reversing the magnetic field ramping direction (see Fig. S8 within the Supplemental Material [21]), where we find no hysteresis behavior of the magnetic-field-dependent $P_{\mathrm{PL}}$. As we show later, to explain and reproduce the unique steplike feature in Fig. 3, one also needs to involve the transverse component of $\mathbf{B}_{N}$ that is tied to some fixed crystallographic direction.

\section{Oblique nuclear quadrupole interaction and its physical origin}

The most likely cause of the observed sizable transverse component of $\mathbf{B}_{N}$ is due to NQI with an oblique principal axis. In a strained QDS, a nucleus with a largespin quantum number $I>1 / 2(I=3 / 2$ for $\mathrm{Ga}$ and As, $I=9 / 2$ for $I n$ ) has a nonvarnishing quadrupole moment that couples to the local electric field gradient (EFG). In the simplest case of axially symmetric EFG, NQI splits the nuclear-spin sublevels into doublets with magnetic quantum number $m= \pm(1 / 2), \pm(3 / 2), \ldots \pm I$. In the low-field regime, NQI dominates over nuclear Zeeman interaction and can strongly modify DNP such that nuclear spins are preferably oriented along the principal axis of NQI. This is the underlying mechanism for stabilization of the nuclear field along the growth direction of symmetric QDs [1]. Generally speaking, the NQI axis of an individual nucleus in a strained QDS can deviate from its growth direction. For each nucleus, we can establish a correspondence between its NQI axis and the measurement frame as depicted in Fig. 4(a). The principal axis of the NQI is marked by its polar and azimuthal angle $\left(\theta_{0}, \phi_{0}\right)\left(0^{\circ}<\right.$ $\theta_{0} \leq 180^{\circ}, 0^{\circ}<\phi_{0} \leq 360^{\circ}$ ). This is true due to the presence of appreciable shear strain in the self-assembled QDs as was suggested by various studies $[17,19,20]$. It is also valid if the effect of alloy randomness is taken into account. As was examined in ( $\mathrm{Al}, \mathrm{Ga})$ As bulk solid solution, by replacing a neighboring $\mathrm{Ga}$ atom by $\mathrm{Al}$, the NQI axis of the As nucleus was found to be along a diagonal $\langle 111\rangle$ direction [26]. It is also suggested that, owing to a strong alloying effect, the NQI axis of an individual nucleus in the (In, Ga)As QDs is determined by its atomic environment. For cations, like In or $\mathrm{Ga}$, one would expect that strain is still the dominant effect as they are surrounded by identical As atoms in the nearest neighbors. However, the situation changes for anions. Taking In composition of $50 \%$ and assuming a homogenous In distribution, we can single out some of the most probable atomic configurations for As atoms, which are displayed in Fig. 4(b). The other two configurations with two In atoms occupying the top or bottom two tetrahedral sites have the principal axis along the [001] direction $\left(\theta_{0}=0\right)$, which cannot cause the observed tilting of the NQI axis of the As atom and are therefore not shown for simplicity. The NQI axes of the As atoms in the four displayed atomic configurations are along $\left(\theta_{0}, \phi_{0}\right)=\left(45^{\circ}, 45^{\circ}\right),\left(135^{\circ}, 135^{\circ}\right),\left(45^{\circ}, 225^{\circ}\right)$, and $\left(135^{\circ}, 315^{\circ}\right)$, respectively [20]. For these nuclei, the 
(a)

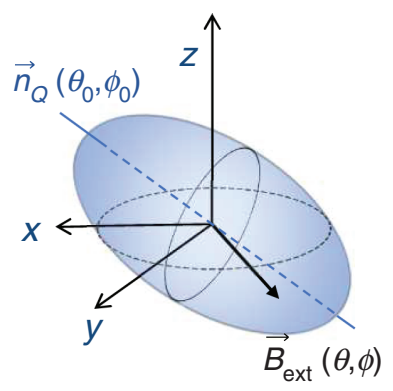

(c)
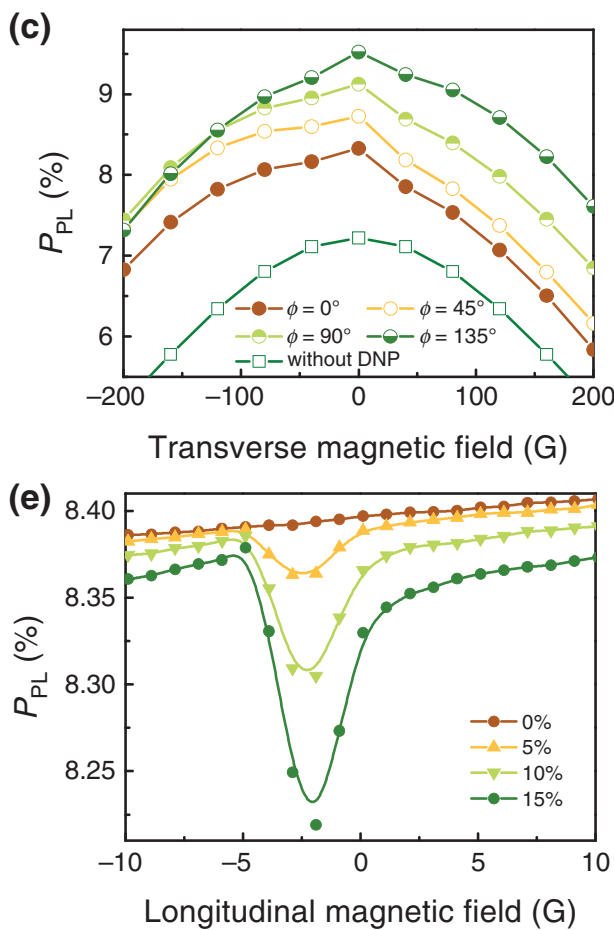

(b)

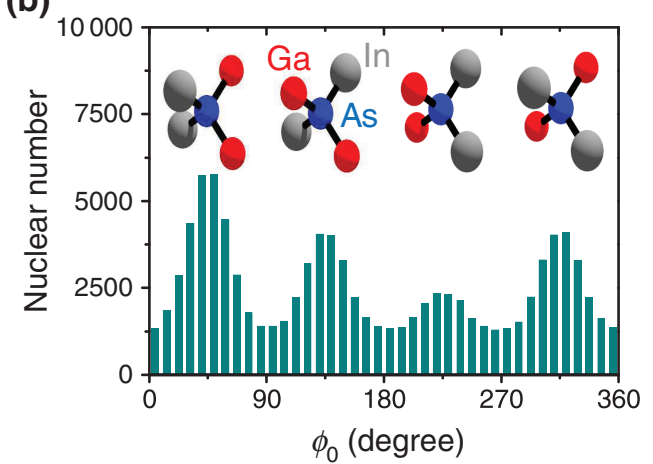

(d)

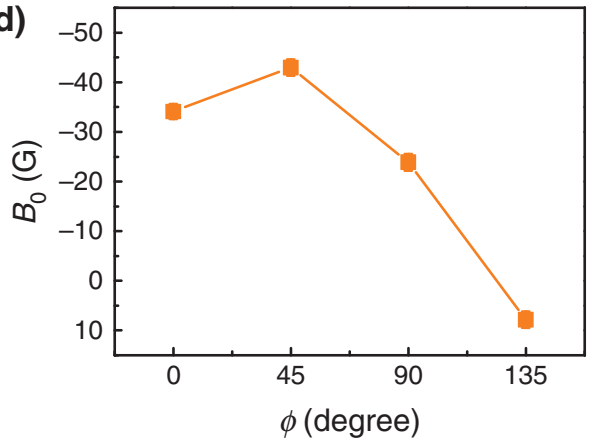

(f)

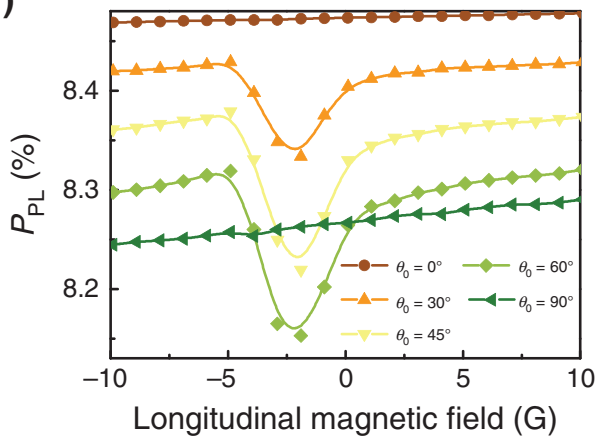

FIG. 4. The theoretical model for oblique NQI and simulations. (a) Illustration of NQI with a tilted principal axis, denoted by $\vec{n}_{Q}$. (b) The atomic configurations and the assumed $\phi_{0}$ distribution of NQI used in calculations. The polar angle is distributed by $50 \%$ around $45^{\circ}$ and $50 \%$ around $0^{\circ}$. In the calculations we assume $15 \%$ imbalance in the preferred atomic configuration. (c) The simulated Hanle curves with different transverse field orientation angles $\phi$. The curves are offset to demonstrate the shift of the broad Hanle component. (d) Angular dependence of $B_{0}$ as determined from the simulated curves in (c). (e), (f) The simulated longitudinal-field dependence with different degrees of imbalance in the atomic configurations (e) and at different tilting polar angles (f), underlining the importance of a partially tilted NQI axis. In (e), the same atomic configuration is used as in (b), but changing the degree of imbalance. In (f), an imbalance of $15 \%$ in the atomic configurations is assumed. $\phi_{0}$ follows the distribution in (b), while the averaged $\theta_{0}$ of the anions changes from $0^{\circ}$ to $90^{\circ}$. nuclear spins are oriented obliquely. DNP in a QDS is a weighted sum of contributions from all the nuclei that are within the electron localization volume. In symmetric structures, this often leads to the vanishing of the above anisotropy, since the effect of different atomic configurations tend to cancel. However, in asymmetric structures like our QDSs, $X^{+}$wave function is expected to be localized within a part of the nanostructures, e.g., an individual dot of the DQD and QC, and In-rich ridge of the QR seen in Fig. 1. To show that such an imbalance in the atomic configurations can be generated and is not constrained due to sharing of cations among the neighboring As atoms, we perform a simulation of a lattice of a lensshaped (In, Ga)As QD embedded in the GaAs host lattice, where formation of the atomic configuration with $\phi_{0}=45^{\circ}$ for As atoms surrounded by two In atoms is promoted. Such an atomic environment in the computer-generated lattice can therefore lead to a net contribution to the tilting of nuclear quadrupolar moment in the QD.
To show that NQI is indeed the reason behind the observed abnormal Hanle behavior, we carry out a detailed rate equation analysis of DNP, including the effect of NQI. In this model, we consider a single electron spin that is closely interacting with $N=100000$ nucleus, which corresponds to the typical size of a single QD. We assume $I=3 / 2$ for all nuclear spins, for simplicity. The calculations for the electron and nuclei are done separately but are interconnected and feedback to each other in the way that the outcome of one is treated as an input parameter of the other (see Appendix A for details). For the electron, we look for the steady state of electron-spin precession in the total field of $\mathbf{B}+\mathbf{B}_{N}$. Here, $\mathbf{B}_{N}=\sum_{i} A^{i} \mathbf{g}_{e}^{-1}\left\langle\hat{\mathbf{I}}_{i}\right\rangle / \mu_{B}+$ $\delta \mathbf{B}_{N}$, which integrates over all the average nuclear spins and includes a fluctuation of nuclear field $\delta \mathbf{B}_{N}$. Here, $\hat{\mathbf{I}}_{i}$ is the nuclear-spin operator for nucleus " $i$ "; $\mu_{B}$ is the Bohr magneton. $\mathbf{g}_{e}$ is the trion $g$ tensor, which has a transverse component $g_{\perp}=-0.8$ and a longitudinal component $g_{\|}=$ 
$-2[27,36]$. The electron spin can then be computed taking all the nuclear spins as input. For a given nuclear spin " $i$ " in return, the Hamiltonian is parameterized by the electron spin, which reads

$$
\begin{aligned}
\hat{H}^{i}= & g_{N}^{i} \mu_{N}\left(\mathbf{B}+\mathbf{B}_{\text {Knight }}\right) \cdot \hat{\mathbf{I}}_{i}+\frac{\Delta_{Q}}{2}\left[\left(\hat{\mathbf{I}}_{i} \cdot \mathbf{n}_{i}\right)^{2}-\frac{I(I+1)}{3}\right] \\
& +\hat{H}_{\text {non }}^{i} .
\end{aligned}
$$

The first terms of $\hat{H}^{i}$ characterize the nuclear Zeeman interaction, which also includes the Knight field effect contributed by the secular part of the electron-nuclear hyperfine interaction. The second term is the NQI in its axial form. Here, $\mathbf{n}_{i}$ is the unit vector along the principal axis of EFG; $\Delta_{Q}$ is the splitting energy of NQI, which is related to the principal value of the EFG tensor $V_{z z}$ and the nuclear quadrupole momentum $Q$ by the relation $\Delta_{Q}=\left[3 e V_{z z} Q / 2 I(2 I-1)\right]$. We note that the EFG and hence NQI can also have axial asymmetry. The biaxiality parameter defined as $\eta=\left(V_{x x}-V_{y y}\right) / V_{z z}$ varies from 0 to 0.6 as shown in Ref. [20]. However, as discussed in detail in Note 2 and Fig. S9 within the Supplemental Material [21], $\eta$ does not lead to the observed magnetoPL signature and is thus set to 0 in our modeling. $\hat{H}_{\text {non }}^{i}=$ $\left(A^{i} / 2\right)\left(\hat{I}_{+} \hat{S}_{-}+\hat{I}_{-} \hat{S}_{+}\right)$represents the nonsecular part of the hyperfine interaction, which is also the driving force for DNP. The nuclear dipole-dipole interaction is not directly included in the Hamiltonian but is reflected by the empirical parameter of the nuclear spin-lattice relaxation time $T_{1}$.

We limit our calculations to the regime $\Delta_{Q} \gg g_{N}^{i} \mu_{N} B$ where the energy spectrum of the nucleus is dominated by the quadrupole splitting between the $|3 / 2, \pm 1 / 2\rangle$ and $|3 / 2, \pm 3 / 2\rangle$ doublets. Here, the nuclear-spin states are labeled as $|I, m\rangle$. The nuclear Zeeman interaction then enters as the first-order perturbation, which splits each doublet and introduces mixing between $|3 / 2,-1 / 2\rangle$ and $|3 / 2,+1 / 2\rangle$ in a general case when the external magnetic field is not parallel to the NQI axis. The DNP is introduced in the usual manner by considering the transition rate $W(i, f)$ from state $\left|\varphi_{i}\right\rangle$ to $\left|\varphi_{f}\right\rangle$ according to Fermi's golden rule [37]:

$$
W(i, f)=\frac{1}{2 T_{1 e}}\left[\left|\left\langle\varphi_{i}\left|\hat{I}_{i+}\right| \varphi_{f}\right\rangle\right|^{2} N_{-}+\left|\left\langle\varphi_{i}\left|\hat{I}_{i-}\right| \varphi_{f}\right\rangle\right|^{2} N_{+}\right] .
$$

Here, $\left|\varphi_{i}\right\rangle$ and $\left|\varphi_{f}\right\rangle$ are the eigenstates of the Hamiltonian excluding the spin flip-flop term [Eq. (1)]. $T_{1 e}$ is the characteristic time of nuclear-electron spin relaxation without the effect of NQI. $N_{ \pm} \equiv 2\left\langle\mathbf{S} \cdot \mathbf{n}_{i}\right\rangle \pm 1$ is the spin-up or spin-down electron population, which takes electron-spin polarization as an input. The population of the nuclear spin sublevels and the corresponding $\mathbf{B}_{N}$ of an individual nuclear spin are governed by the rate equation described in Appendix A. $T_{1 e}$ is set to $100 \mu \mathrm{s}$ based on the measured DNP build-up time from the time-resolved measurement shown in Fig. S10 within the Supplemental Material [21]. We should note that $T_{1 e}$ determined here is much smaller compared to the earlier reports on (In, Ga)As QDs by detecting the negative charge exciton $X^{-}$emission $[38,39]$. For rough estimation, $T_{1 e} \sim \Omega_{e}^{2} \tau_{\mathrm{el}} /(A / N \hbar)^{2}$ is usually used, which is originally derived for nuclearspin depolarization in the presence of a localized defect electron. Here $A$ is the sum of the hyperfine constant of the unit cell nuclei, $N$ is the number of nuclei within the localization volume of the electron, $\Omega_{e}$ is the electron-spin splitting, and $\tau_{\mathrm{el}}$ is the correlation time of electron-spin fluctuation. The measurement on $X^{-}$monitors the nuclearspin interaction with the residual electron in the ground state, where $\tau_{\text {el }}$ is only limited by the spin-flip time on the order of $20 \mathrm{~ns}$. This is different from our case where the hyperfine interaction takes place between the nuclear spins and the electron spin in the $X^{+}$state, which is limited by the $X^{+}$lifetime on the order of a few hundred ps. As a consequence, the measured $T_{1 e}$ from Fig. S10 within the Supplemental Material is much shorter. The dynamic equation of coupled electron and nuclear spins is solved from time $t=0$ to $500 \mu \mathrm{s}$ when the DNP is complete and the steady state of electron- and nuclear-spin polarization is reached. For each time step of $30 \mathrm{~ns}$, we update the states of nuclear spins and calculate the electron-spin polarization using the newly configured nuclear spins. At the initial time $t=0$, an equal population of nuclear sublevels is assumed and the corresponding field dependence can be compared to the experimental results without DNP. The results at $t=500 \mu \mathrm{s}$, on the other hand, represent the simulations including the effect of DNP.

Figures 4(c)-4(f) show the simulation results, using the aforementioned atomic configurations. Here, we mainly consider the effect of atomic configurations, but bear in mind that the strain effect can be addressed in the same way by assuming a configuration with $\mathbf{n}_{i}$ along the principal axis of the strain tensor. For cations, $\theta_{0}$ is set to be around $0^{\circ}$ and $\phi_{0}$ is distributed uniformly between $0^{\circ}$ and $360^{\circ}$. For anions, the four atomic configurations displayed in the inset of Fig. 4(b) are used and $\left(\theta_{0}, \phi_{0}\right)$ is distributed around the corresponding principal directions. The angular distribution is artificially broadened to accommodate the effect of strain relaxation. The distribution of $\phi_{0}$ after considering $15 \%$ imbalance in the anion atomic configurations is plotted in Fig. 4(b). Taking this atomic configuration as input, as shown in Fig. 4(c), we can more or less quantitatively reproduce the Hanle curve shown in Fig. 2(a). The simulated curves show a sharp peak located at zero field and a broad component that shifts its peak position as we change field orientation $\phi$. The narrow component is better seen in the simulation in the narrow-field range in Fig. S11 within the Supplemental Material [21]. As plotted in Fig. 4(d), the largest $B_{0}$ is achieved when the applied field is oriented 
along the NQI axis of the preferable atomic configuration. Using the same simulation setup, we can also reproduce the anomalies in the Faraday geometry, which is shown in Figs. 4(e) and 4(f). In Fig. 4(e), we vary the imbalance of the atomic configurations from $0 \%$ to $15 \%$ and simulate the corresponding $P_{\mathrm{PL}}$ in the low-field regime. It shows that, with a gradually increasing imbalance of the distribution, the steplike feature develops at the compensation point of $\mathbf{B}_{\text {Knight. In the simulation over a wider field range }}$ as shown in Fig. S12 within the Supplemental Material [21], we show that the features of $\mathbf{B}_{\text {Knight }}$ and $\mathbf{B}_{N}$ are comparable to the experimental results shown in Fig. 3(a). In Fig. 4(f), we demonstrate that such a phenomenon is only associated with the atomic configurations that are oblique with respect to the $z$ direction. Keeping the same degree of imbalance (15\%), the "abnormal" spectrum only occurs at $\theta_{0}=30^{\circ}, 45^{\circ}$, and $60^{\circ}$, whereas the "regular" shape is observed at $0^{\circ}$ and $90^{\circ}$.

The fact that both transverse- and longitudinal-field anomalies can be derived from the same theoretical model with the same set of parameters confirms the build up of transverse OHF due to the oblique NQI in the asymmetric QDSs. The anomalous magneto-optical feature discovered in this work does not change with the field scanning directions (see Fig. S8 within the Supplemental Material [21]), in sharp contrast to the previously reported effect of transverse OHF in a single QD that leads to an anomalous Hanle curve with a broad peak and optical bistability when the magnetic field scanning direction is reversed $[11,40]$. We note that the transverse OHF in these early reports stems from magnetic-field-induced symmetry breaking and vanishes in the absence of $B_{\perp}$. This is markedly different from our case in which the transverse OHF can be built up without an applied magnetic field. Additionally, as pointed out in Ref. [40], strong anisotropy of a nuclear $g$ factor, to the extent that longitudinal and transverse $g$ factor have different signs, is required for the observation of the anomalous Hanle effect. In contrast, our simulation indicates that the magneto-optical anomalies observed in this work does not have such a requirement. The distinct differences between this work and earlier results on anomalous Hanle effect suggest a unique and previously unseen role of the oblique NQI that is inherent in our asymmetric QDSs, even without an external symmetry-breaking magnetic field.

\section{E. Influence from the lateral arrangement of the QDSs}

The lateral arrangement of the QDSs is shown to have a strong influence on the tilting of NQI. This is firstly seen from the absence of the corresponding magneto-optical anomalies from the strained single-QD (SQD) sample, which is shown in Fig. S13 within the Supplemental Material in both Faraday and Voigt geometry [21]. In Fig. 2(d), we also include the $B_{0}$ values extracted from the measurements on the SQD sample. Although comparable $B_{N}^{L}$ is observed in Fig. S13(e) within the Supplemental Material, the transverse OHF component $B_{N}^{T}(\phi)$ vanishes at all probed angle $\phi$. This suggests that in the absence of the laterally surrounding QD, no effect of oblique NQI can be detected. Separating the effect of shear strain and the alloying atomic configuration is difficult as one would expect that the strain tensor and the overall atomic configuration are interconnected such that a tilted strain axis favors the formation of certain atomic configurations. This is further demonstrated in Fig. S14 within the Supplemental Material, where generation of imbalance in the immediate atomic environment of As atoms is shown to lead to a net shear strain in a simulated lattice of an (In, Ga)As quantum $\operatorname{dot}[21]$. Nevertheless, we find that the lateral arrangement of the QDSs affects strain and/or alloying atomic configuration that leads to observation of a consistent effect on the angular dependence of $B_{0}$ as well as the statistics of fine-structure splitting in individual QDS throughout the investigated samples, as presented below.

\section{Angular dependence of $B_{0}$}

The angular dependence of $B_{0}$ shown in Fig. 2(d) reveals the strength and preferable orientation of the transverse $\mathrm{OHF}$ and thus reflects the distribution of in-plane projection of the oblique NQI axis in different QDSs. In the QC sample, $B_{0}(\phi)$ has a relatively large value and a single preferable direction at around $\phi=90^{\circ}$. In the case of the DQD sample, however, $B_{0}(\phi)$ has a much lower value and has two preferable orientations identified at $\phi=45^{\circ}$ and $135^{\circ}$. We note that, in the latter case, the preferable orientations coincide with the NQI axis of the two atomic configurations that we introduce in Fig. 3(b), which may indicate the involvement of the effect of the alloying atomic configuration. The same angular behavior is also observed in the QR sample, but the overall strength of $B_{0}$ is much stronger than DQDs.

\section{Effect on the AEI}

Due to the AEI, the bright $X^{0}$ exciton state of a lowsymmetry QD splits into two states, namely $|X\rangle$ and $|Y\rangle$, which give rise to linearly polarized emissions. The polarization directions of the doublet emissions are marked by the dashed lines as shown in Fig. 5(a) for an individual DQD. $\theta_{X^{0}}$ is defined as the angle between the two polarization directions. Typically, $|X\rangle$ and $|Y\rangle$ has orthogonal polarization and $\theta_{X^{0}}=90^{\circ}$. The shear-strain components $\varepsilon_{x z}$ and $\varepsilon_{y z}$, i.e., the same components responsible for the oblique NQI, can mix the exciton states in the way that the doublet emission is no longer orthogonal (i.e., $\left.\theta_{X^{0}}<90^{\circ}\right)$. This is seen in Fig. 5(b), where we simulate the $\theta_{X^{0}}$ as a function of the shear-strain component $\varepsilon_{s} e^{i \gamma}=\varepsilon_{x z}-i \varepsilon_{y z}$ following the effective Hamiltonian described in Appendix B. We find that the $\theta_{X^{0}}$ deviates 

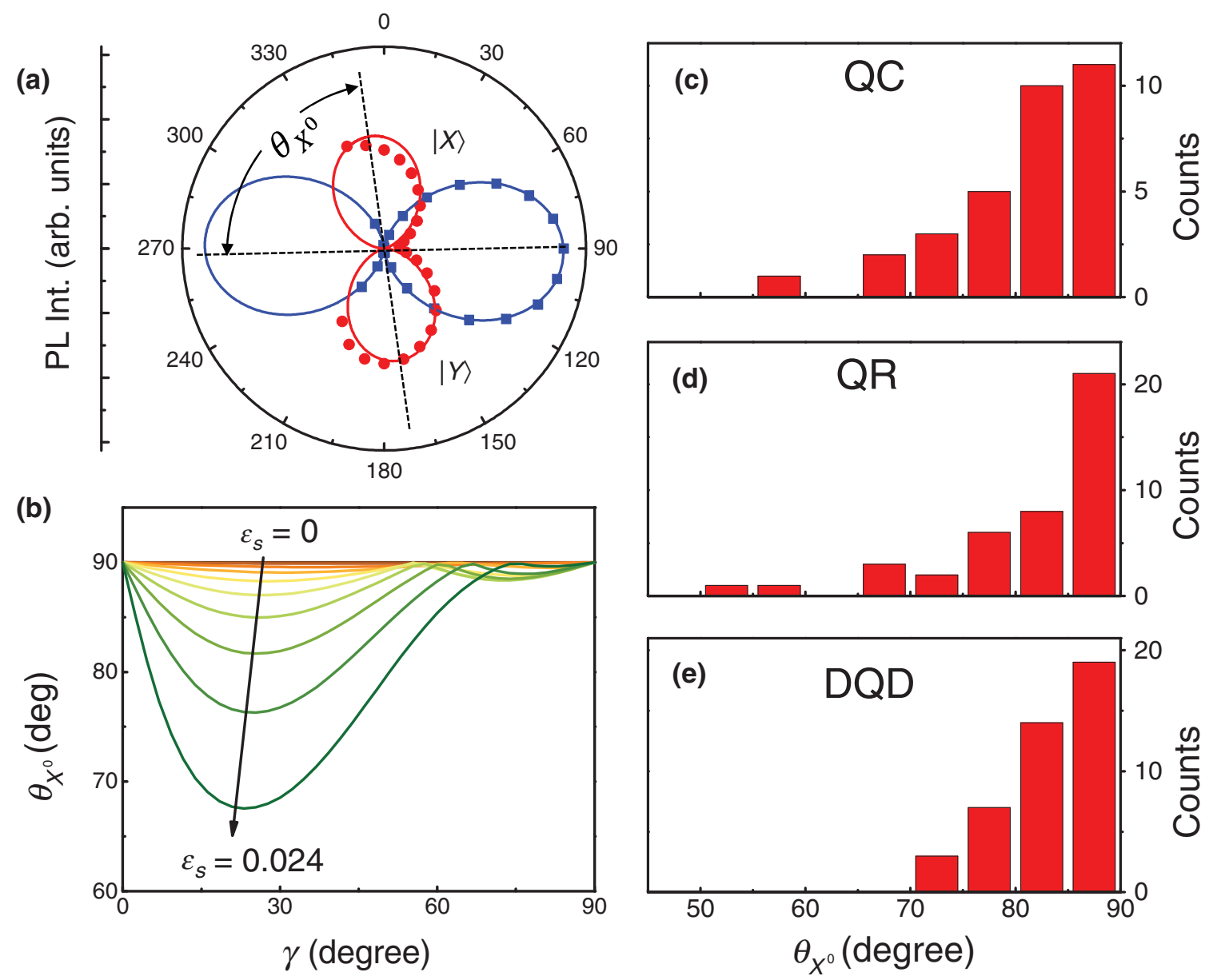

FIG. 5. Estimation of shear strain from the statistics of AEI. (a) Illustration the PL intensity from the two bright $X^{0}$ states measured from a single DQD as a function of rotation angles of the linear-polarization analyzer. The angle $\theta_{X^{0}}$ between the polarization directions of the doublet emission is marked. (b) The simulated $\theta_{X}$ with different values of the shear-strain component $\varepsilon_{s} e^{i \gamma}=\varepsilon_{x z}-i \varepsilon_{y z}$. The statistical counts of $\theta_{X^{0}}$ measured from individual QC (c), QR (d), and DQD (e) are also shown. $\theta_{X^{0}}$ spreads out to lower values in the QCs and QRs as compared to the DQDs, indicating a more pronounced shear strain in the former structures. The data are obtained at $5 \mathrm{~K}$ by a single-dot PL spectroscopy with unpolarized excitation above the GaAs bandgap.

from $90^{\circ}$ when the shear-strain component increases. Since it is unlikely that the short-range alloying configuration will have any noticeable effect on the exciton states, this effect is only sensitive to the strain condition and can be used to disentangle the effect of strain and alloying. In Figs. 5(c)-5(e), we present the statistics of the $\theta_{X^{0}}$ extracted from the QCs, QRs, and DQDs samples, respectively. The measurements are performed at a cryogenic temperature on over 30 individual QDSs for each sample. The $\theta_{X^{0}}$ in the QC and QR samples are found to be distributed towards lower values as compared to that in the DQD sample, suggesting a weaker effect of shear strain in DQD. This is in line with observation of overall much weaker $B_{0}$ in DQDs. One can roughly estimate the shear-strain strength in the nanostructure from the lower boundary of the $\theta_{X^{0}}$ distribution. With the simulation parameter in Appendix B and considering a lower boundary of about $\theta_{X^{0}}=65^{\circ}$ as in the QC sample, the magnitude of shear strain is about 0.024 . We note this is a quite large amount and can lead to a tilting angle of the NQI as large as $20^{\circ}$, which is comparable with the value we deduce from the OHF and thus suggests a rather important role of the strain effect.

The two experimental results basically show a consistent picture that the lateral arrangement of the QDSs affects the tilting of NQI and build up of the transverse OHF by modifying the strain and/or alloying configurations of the nanostructures. The contribution of the shear strain is found to be much stronger in the QR and QC samples than DQDs, while angular dependence of $B_{0}$ suggests that the alloying configurations may also play a role in the DQD sample.

From the magnitude of the transverse component of the OHF and the nonorthogonality of the bright exciton doublet, one can observe a trend on the degree of symmetry breaking of the QDSs. To demonstrate this, we summarize 
TABLE I. Summary of the experimental values obtained from the quantum-dot structures studied in this work.

\begin{tabular}{lcccc}
\hline \hline Sample & $\left|B_{0}^{\max }\right|(\mathrm{G})$ & $B_{N}^{L}(\mathrm{G})$ & $\phi_{0}$ & $\left|\theta_{X^{0}}-90^{\circ}\right|$ \\
\hline QCs & 100.2 & 269 & $90^{\circ}$ & $9.1^{\circ}$ \\
QRs & 72.1 & 335 & $45^{\circ}$ & $8.1^{\circ}$ \\
DQDs & 30.9 & 344 & 0 & $6.6^{\circ}$ \\
SQDs & 3.6 & 460 & $45^{\circ}$ & - \\
\hline \hline
\end{tabular}

the $\left|B_{0}^{\max }\right|, B_{N}^{L}$, and $\left|\theta_{X^{0}}-90^{\circ}\right|$ for each of the studied samples in Table I, where $\left|B_{0}^{\max }\right|$ and $B_{N}^{L}$ are the maximum transverse OHF and the longitudinal OHF probed in the measurements with transverse and longitudinal magnetic field, respectively. $\left|\theta_{X^{0}}-90^{\circ}\right|$ measures the mean deviation of $\theta_{X^{0}}$ from $90^{\circ}$. On one hand, for symmetry of $C_{2 v}$ or higher, $B_{0}$ vanishes following the symmetry consideration given in Note 2 within the Supplemental Material [21]. The observation of pronounced $B_{0}^{\max }$ in the $\mathrm{DQD}, \mathrm{QR}$, and QC as compared to the SQD sample, despite the fact that comparable or even stronger $B_{N}^{L}$ is observed in the latter, thus suggests a more severe symmetry-breaking effect in the laterally arranged QDs. Additionally, the largest $B_{0}^{\max }$ is found in QC whereas the smallest $B_{0}^{\max }$ is seen in DQD. On the other hand, the reduction of symmetry due to the presence of shear strain leads to the nonorthogonality of the bright exciton states, which can qualitatively be estimated with $\left|\theta_{X^{0}}-90^{\circ}\right|$. In Table I, $\left|\theta_{X^{0}}-90^{\circ}\right|$ exhibits a similar trend as $B_{0}^{\max }$, which suggests an increased symmetrybreaking effect in QC as compared to DQD. These two approaches, which are derived independently from the excitonic and spintronic effect of the QDSs, thus provide reliable assessment of the symmetry reduction of the QDSs and offer alternative perspectives and tools for studies of low-symmetry nanostructures.

One consequence of the oblique NQI is insufficient polarization of the electron and nuclear spins. In Fig. 6, we simulate the averaged electron and nuclear spin polarization in the absence of a magnetic field as the average NQI is tilted from $\theta_{0}=0^{\circ}$ to $90^{\circ}$, while $\phi_{0}$ is kept at around $45^{\circ}$. As the principal axis of NQI is tilted away from the $z$ direction, the nuclear-spin polarization reduces monotonously. This is mainly due to an inefficient DNP with increasing $\theta_{0}$, since only the electron-spin projection onto the NQI axis actually contributes to DNP. The electron-spin polarization first decreases and then increases when $\theta_{0}$ increased from $0^{\circ}$ to $90^{\circ}$. We stress here that the initial decrease of $\left\langle S_{z}\right\rangle$ from $\theta_{0}=0^{\circ}$ to $50^{\circ}$ mainly originates from the presence of transverse OHF. The transverse OHF causes the electron spin to precess around an oblique axis and eventually leads to dephasing and decline of electron-spin polarization in the QDS ensembles. Such an effect is in addition to the commonly addressed spin-dephasing effect from the random-oriented NQI and should play an important role in low-symmetry nuclear-spin-rich QDSs [13]. Further increase of $\theta_{0}$ leads to inefficient DNP, which reduces both

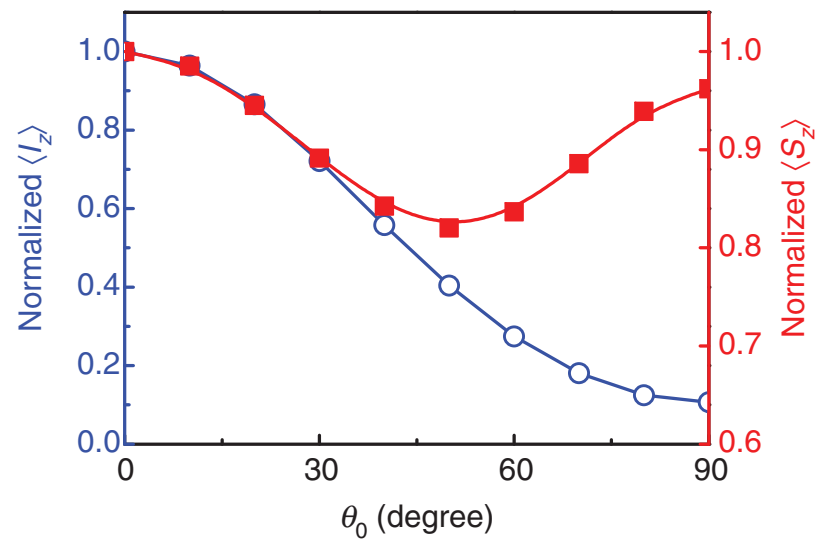

FIG. 6. Electron- and nuclear-spin polarization versus tilting angle of the nuclear quadrupole interaction axis. Simulated averaged electron $S_{z}$ and nuclear $I_{z}$ spin polarization at zero external magnetic field as a function of $\theta_{0}$ - the averaged tilting angle of the quadrupole axis from the $z$ direction. Both $S_{z}$ and $I_{z}$ are normalized to their values at $\theta_{0}=0$.

the longitudinal and transverse component of OHF. This releases the electron spin from dephasing caused by the transverse OHF and recovers $\left\langle S_{z}\right\rangle$. We should note that $\left\langle S_{z}\right\rangle$ at $\theta_{0}=90^{\circ}$ is lower than the value at $\theta_{0}=0^{\circ}$. This is reasonable, as DNP starts to fade away, the randomly oriented fluctuating OHF $\delta \mathbf{B}_{N}$ dephases the electron spin. In a direct comparison between $\left\langle S_{z}\right\rangle$ at $\theta_{0}=0^{\circ}, 50^{\circ}$, and $90^{\circ}$, one can see that the obliquely oriented OHF can cause higher spin loss as compared to the case without DNP, which highlights the importance of fine tuning of QDS growth to avoid the oblique NQI. However, a direct measurement of the simulated $\theta_{0}$ dependence in Fig. 6 is not feasible here, as it requires continuously changing $\theta_{0}$ with carefully modified strain or alloying condition and at the same time keeping other conditions unaffected. Nevertheless, the proposed mechanism will be in effect when the tilting of the NQI is detected, for instance, as in the QDSs studied in this work. The simulations thus give a guideline on how much the electron and nuclear spin polarization is affected.

We notice that, in self-assembled (In, Ga)As QDSs, the implementation of strain engineering and control of In composition to produce "better" QDSs have attracted intense studies during recent decades. For instance, it was previously shown that, by compensating the QD shape anisotropy by strain, the most "symmetric" emitters can be achieved in the QC sample, where the fine-structure splitting of $X^{0}$ was minimized [41]. However, the results shown in Fig. 2(d) indicate that the QC sample is not "symmetric" at all in terms of nuclear-spin environment as it gives rise to the largest asymmetry in the Hanle curve. Such opposite trends in electron and nuclear spins demonstrate that the electrostatic environments felt by the nuclei are very different from the confined excitons. A highsymmetry confinement potential for the neutral excitons 
does not guarantee a high-symmetry NQI for nuclei within the same nano-object. In contrast, it may enhance the transverse nuclear field that promotes electron-spin decoherence and depolarization. This calls for caution that the optimization in the symmetry of the electronic confinement potential and that of the nuclear-spin bath needs to be carried out hand in hand or differently depending on specific applications.

\section{CONCLUSION}

In summary, we present our detailed magneto-optical studies of a series of laterally arranged (In, Ga)As nanostructures including DQDs, QCs, and QRs in the context of DNP. We show that the field dependence of electronspin polarization significantly deviates from the commonly seen behavior of QDs in both Voigt and Faraday geometries. We find that the observed anomalies appear only under the condition when DNP is present and its behavior can be understood by assuming a transverse component of the Overhauser field that is fixed to a certain crystallographic orientation. We suggest the presence of an oblique NQI, due to the imbalance of shear strain or/and alloy randomness in the low-symmetry QDSs, as the underlying mechanism for the observation. We show that, by taking into account the oblique NQI, we can quantitatively reproduce the magneto-optical spectra in both Voigt and Faraday geometries with the same set of simulation parameters. Within this model, we are able to determine the distribution of the in-plane projection of the oblique NQI axis and a direct detrimental effect of such tilting NQI in the electron- and nuclear-spin polarization. The oblique NQI discovered in these QDSs may have a strong impact on the pursuit of quantum entanglement and scaling up of the quantum-information system, which are routinely explored in closely packed and strongly coupled QDs $[42,43]$. Moreover, with the need for increasing information storage density and processing speed in future spintronic and quantum-information systems, the dot-to-dot distance in QD-based systems may essentially come to the limit where a long-range interaction of strain between the adjacent QDs will play an important role in the electron-nuclear spin interactions and spin depolarization. To meet these application challenges, this work calls for future efforts that aim to simultaneously optimize the symmetries of the electronic and spin environments experienced by the confined electrons, excitons, and nuclear spin bath, such that all major spin decoherence and depolarization processes can be suppressed, which is essential to future applications of these semiconductor nanostructures in spintronics and quantum-information technology.

\section{ACKNOWLEDGMENTS}

We are grateful to C.W. Tu for providing the samples. This work is supported by Linköping University through the Professor Contracts, the Swedish Research Council (Grant No. 2016-05091), and the Swedish Government Strategic Research Area in Materials Science on Functional Materials at Linköping University (Faculty Grant SFO-Mat-LiU No. 2009-00971).

\section{APPENDIX A: RATE EQUATION ANALYSIS OF ELECTRON-NUCLEAR SPIN DYNAMICS}

In this model, we consider dynamics of a single electron spin subjected to hyperfine interactions with $N=100000$ nuclear spins. The full Hamiltonian can be written as follows:

$$
\begin{aligned}
\hat{H}= & \mu_{B} \mathbf{B} \cdot \mathbf{g}_{e} \hat{\mathbf{S}}+\sum_{i}\left\{g_{N} \mu_{N} \mathbf{B} \cdot \hat{\mathbf{I}}_{i}+\frac{\Delta_{Q}}{2}\left[\left(\hat{\mathbf{I}}_{i} \cdot \mathbf{n}_{i}\right)^{2}\right.\right. \\
& \left.\left.-\frac{I(I+1)}{3}\right]+\hat{H}_{e-n}^{i}\right\} .
\end{aligned}
$$

It includes, from left to right in Eq. (A1), the Zeeman interaction terms for both electron and nuclear spins, nuclear quadrupole interaction, as well as coupling terms between electron and nuclear spin. Here, $\mathbf{g}_{e}$ and $\mu_{B}$ are the trion $g$ tensor and Bohr magneton. We take the transverse and longitudinal components of $\mathbf{g}_{e}$ to be $g_{\perp}=-0.8$ and $g_{\|}=-2$, which are reported in the literature. We should note that a slight change of the $g$ factor does not affect our qualitative conclusion drawn from the simulations [27,36]. $g_{N}$ and $\mu_{N}$ are the corresponding nuclear $g$ factor and nuclear magneton, which are taken to be the same for all the nuclei for simplicity. $\hat{\mathbf{S}}$ and $\hat{\mathbf{I}}_{i}$ are the electron-spin and nuclear-spin operator. $\Delta_{Q}$ represents the nuclear quadrupole splitting. $\mathbf{n}_{i}$ is the unit vector denoting the principal axis of the nuclear quadrupole interaction. The electron-nuclear spin interaction is in the form of the hyperfine interaction,

$$
\hat{H}_{e-n}^{i}=A_{i} \hat{I}_{z^{\prime}} \hat{S}_{z^{\prime}}+\frac{A_{i}}{2}\left(\hat{I}_{i+} \hat{S}_{-}+\hat{I}_{i-} \hat{S}_{+}\right),
$$

with $\hat{I}_{i z^{\prime}}$ and $\hat{S}_{z^{\prime}}$ being the nuclear and spin projection operator along the direction defined by $\mathbf{n}_{i}$. The hyperfine tensor is assumed to be isotropic. The spin flip-flop term defines the transfer of angular momentum between the electron and nucleus, which serves as the driven force for dynamic nuclear polarization in our system. To simulate the dynamics with the above Hamiltonian and the corresponding magneto-optical behavior, we consider the case of an electron spin interacting with nuclear spins with $I=3 / 2$ in the framework of a mean-field approximation, where the electron spin evolves in the external magnetic field and an effective time-varying Overhauser field. The time-dependent effective field $\mathbf{B}_{e}^{\text {eff }}(t)$ for the electron spin 
takes the form,

$$
\mathbf{B}_{e}^{\mathrm{eff}}(t)=\mathbf{B}+\sum_{i} A_{i} \mathbf{g}_{e}^{-1}\left\langle\hat{\mathbf{I}}_{i}\right\rangle_{t} / \mu_{B}
$$

Here, $\left\langle\hat{\mathbf{I}}_{i}\right\rangle_{t}$ represents the expected value of the nuclear spin of the $i$ th nucleus evaluated at time $t$. For the electron, the effective form of the Hamiltonian reads

$$
\hat{H}_{e}^{\mathrm{eff}}=\mu_{B} \mathbf{B}_{e}^{\mathrm{eff}}(t) \cdot \mathbf{g}_{e} \hat{\mathbf{S}} .
$$

The Hamiltonian describes an energy conserved system where the electron spin undergoes precession in the total effective magnetic field. The effect of nuclear spins appears as a time-dependent parameter that needs to be updated at each point in a time sequence. In reality, not only is the electron spin optically oriented and being captured by the QD regularly, but it also can be annihilated due to recombination with holes or a spin-relaxation process that happens within the exciton lifetime $\tau_{\text {lifetime }}$ and spin-flip time $T_{s}$. To include the generation and annihilation term, the dynamics of the electron spin can be captured in a semiclassical fashion with the Bloch equation:

$$
\frac{d\left\langle\hat{\mathbf{S}}_{t}\right\rangle}{d t}=\mathbf{B}_{e}^{\mathrm{eff}}(t) \times\langle\hat{\mathbf{S}}\rangle_{t}-\frac{\langle\hat{\mathbf{S}}\rangle_{t}}{T_{s}}-\frac{\langle\hat{\mathbf{S}}\rangle_{t}-S_{0}}{\tau_{\text {lifetime }}} .
$$

Since the motion of the electron spin is expected to be much faster than the nuclear-spin dynamics, the electron spin can be regarded as always being in the steady state of Eq. (A5), which can be solved analytically when $\left\langle\hat{\mathbf{I}}_{i}\right\rangle_{t}$ is provided for every nucleus.

To treat the dynamics of the nuclear spins, one needs to consider the effect of DNP, which is the key condition of the experimental observation. Since we do not explicitly include nuclear spin-spin interactions, we can address each nuclear spin independently. In this simplified picture, we consider a time-dependent perturbation with a reduced Hamiltonian for the $i$ th nucleus:

$$
\hat{H}_{N}^{i}=\hat{H}_{0}^{i}+\frac{A_{i}}{2}\left(\hat{I}_{i+} \hat{S}_{-}+\hat{I}_{i-} \hat{S}_{+}\right)_{t} .
$$

Here $\hat{H}_{0}^{i}=g_{N} \mu_{N} \mathbf{B}_{N}^{\mathrm{eff}} \cdot \hat{\mathbf{I}}_{i}+\left(\hbar \omega_{Q}^{i} / 2\right)\left\{\left(\hat{\mathbf{I}}_{i} \cdot \mathbf{n}_{i}\right)^{2}-[I(I+1)] /\right.$ $3\}$ is the unperturbed Hamiltonian, which contains a nuclear Zeeman interaction term with an effective field $\mathbf{B}_{N}^{\text {eff }}=\mathbf{B}+\mathbf{n}_{i} \cdot \eta A_{i} \hat{S}_{z^{\prime}} / g_{N} \mu_{N}$ and the nuclear quadrupole interaction. $\eta$, with $0<\eta<1$, is the parameter describing the occupation probability of the quantum dot. We note that the electron-nuclear spin hyperfine interaction (HFI) are divided into two parts. The first part is the electronnuclear spin flip-flop term contributed by the off-diagonal part of the HFI. It serves as a time-dependent perturbation, which is the driven force of DNP. The second part is the diagonal contribution of the HFI, contributing to $\mathbf{B}_{N}^{\text {eff }}$. Similar to the case of the electron, $\mathbf{B}_{N}^{\text {eff }}$ depends on the steady-state electron-spin projection along the quadrupole axis, namely $\left\langle\hat{S}_{z^{\prime}}\right\rangle$. We consider the case where the nuclear quadrupole interaction dominates over the nuclear Zeeman and the hyperfine interaction $\left(\hbar \omega_{Q}^{i} / g_{N} \mu_{N} \gg\left|\mathbf{B}_{N}^{\text {eff }}\right|\right)$, which is expected in strained quantum dots. According to $\hat{H}_{0}^{i}$, the energy level of a nucleus with $I_{i}=3 / 2$ splits into four nondegenerated levels with their eigen energies

$$
\begin{aligned}
E_{ \pm \frac{3}{2}} & =\Delta_{Q} \pm \frac{3}{2} \Delta_{z m} \cos \vartheta, E_{ \pm \frac{1}{2}} \\
& = \pm \frac{1}{2} \Delta_{z m} \sqrt{\cos ^{2} \vartheta+4 \sin ^{2} \vartheta}
\end{aligned}
$$

and the corresponding eigen states

$$
\left|\varphi_{ \pm \frac{3}{2}}\right\rangle=| \pm 3 / 2\rangle,\left|\varphi_{ \pm \frac{1}{2}}\right\rangle=\alpha_{ \pm 1 / 2}|+1 / 2\rangle+\beta_{ \pm 1 / 2}|-1 / 2\rangle .
$$

Here, we use the basis set of $| \pm 1 / 2\rangle$ and $| \pm 3 / 2\rangle$, which are the nuclear spin states with $I_{i z^{\prime}}= \pm(1 / 2)$ and $\pm(3 / 2)$. $\Delta_{Q}$ is the quadrupole splitting. $\Delta_{\mathrm{Zm}}$ is the nuclear Zeeman splitting, which scales with the strength of the effective field $\left|\mathbf{B}_{N}^{\text {eff }}(t)\right| . \vartheta$ is the angle between $\mathbf{n}_{i}$ and $\mathbf{B}_{N}^{\text {eff }}$. The hyperfine flip-flop term initiates the transition between the nuclear sublevels with the Fermi-Golden-type transition rate,

$$
W(i, f)=\frac{1}{2 T_{1 e}}\left[\left|\left\langle\varphi_{i}\left|\hat{I}_{i+}\right| \varphi_{f}\right\rangle\right|^{2} N_{-}+\left|\left\langle\varphi_{i}\left|\hat{I}_{i-}\right| \varphi_{f}\right\rangle\right|^{2} N_{+}\right] .
$$

Here, $1 / T_{1 e}$ is the characteristic rate of electron-nuclear spin flip flop. $N_{ \pm} \equiv 1 \pm 2\left\langle\mathbf{S} \cdot \mathbf{n}_{i}\right\rangle$ is the spin-up or spindown electron population, which is evaluated at each point in the time sequence. The matrix element $\left|\left\langle\varphi_{i}\left|\hat{I}_{i \pm}\right| \varphi_{f}\right\rangle\right|^{2}$ can be directly calculated from the eigen basis in Eq. (A8). In the presence of finite spin polarization along the nuclear quadrupole axis (for instance, $N_{+}>N_{-}$), $W(i, f)$ promotes the transition from the state with the lower nuclear spin projection to the higher ones that leads to build up of DNP. The process can be described with the aid of the following coupled rate equations,

$$
\begin{aligned}
\frac{d n_{ \pm 3 / 2}}{d t}= & -\left[W\left( \pm \frac{3}{2}, \frac{1}{2}\right)+W\left( \pm \frac{3}{2},-\frac{1}{2}\right)\right] n_{ \pm 3 / 2} \\
& +W\left(\frac{1}{2}, \pm \frac{3}{2}\right) n_{1 / 2}+W\left(-\frac{1}{2}, \pm \frac{3}{2}\right) n_{-1 / 2} \\
& -\frac{n_{ \pm 3 / 2}}{T_{1}}
\end{aligned}
$$




$$
\begin{aligned}
\frac{d n_{ \pm 1 / 2}}{d t}= & -\left[W\left( \pm \frac{1}{2}, \frac{3}{2}\right)+W\left( \pm \frac{1}{2},-\frac{3}{2}\right)\right. \\
& \left.+W\left( \pm \frac{1}{2}, \mp \frac{1}{2}\right)\right] n_{ \pm 1 / 2}+W\left(\mp \frac{1}{2}, \pm \frac{1}{2}\right) n_{\mp 1 / 2} \\
& +\left(-\frac{3}{2}, \pm \frac{1}{2}\right) n_{-3 / 2}+W\left(\frac{3}{2}, \pm \frac{1}{2}\right) n_{3 / 2}-\frac{n_{ \pm 1 / 2}}{T_{1}}
\end{aligned}
$$

Here, $n_{ \pm 1 / 2}$ and $n_{ \pm 3 / 2}$ are the populations of the corresponding nuclear sublevels in Eqs. (A7) and (A8). To account for the nuclear-spin relaxation due to nuclear spin-spin and spin-lattice interaction, we also introduce an effective relaxation time of $T_{1}$. Equations (A10.1) and (A10.2) give the evolution of the nuclear-spin sublevel populations, which can be used to determine $\left\langle\hat{\mathbf{I}}_{i}\right\rangle_{t}$ for the $i$ th nucleus at time $t$ through the relation, $\left\langle\hat{\mathbf{I}}_{i}\right\rangle_{t}=$ $\sum_{\xi= \pm 1 / 2, \pm 3 / 2} n_{\xi}\left\langle\varphi_{\xi}\left|\hat{\mathbf{I}}_{i}\right| \varphi_{\xi}\right\rangle$.

We firstly need to assume a distribution of $\mathbf{n}_{i}$, which determines the principal direction of the nuclear quadrupole moment for each nucleus. In accordance with the $T_{d}$ symmetry of the lattice and previous calculations [20], we divide the anion nuclei into four ensembles with the averaged principal axis $\overline{\mathbf{n}_{i}}$ of each pointing along the four directions with the polar and azimuth angles $\left(\theta_{0}, \phi_{0}\right)=\left(45^{\circ}, 45^{\circ}\right),\left(135^{\circ}, 135^{\circ}\right),\left(45^{\circ}, 225^{\circ}\right)$, and $\left(135^{\circ}, 315^{\circ}\right)$. While the $\overline{\mathbf{n}_{i}}$ of the cation nucleus is assumed along the $z$ direction $\theta_{0}=0^{\circ}$. Within each ensemble, the angle between $\mathbf{n}_{i}$ and $\overline{\mathbf{n}_{i}}$ follows a normal distribution with a standard deviation of $15^{\circ}$. The preferential orientation of the nuclear quadrupole axis can then be controlled by taking different ratios between the four ensembles.

The full calculations involving 100000 nuclear spins with $I=3 / 2$ are performed iteratively through the time sequence $t=0, \Delta t, 2 \Delta t, \cdots$. The iteration starts by assuming equally populated nuclear sublevels at $t=0$. As a consequence, $\left\langle\hat{\mathbf{I}}_{i}\right\rangle_{t=0}=0$ for all nuclei. Between each time interval from $t$ to $t+\Delta t$, the calculations are carried out iteratively. For each nuclear spin, the unperturbed Hamiltonian in Eq. (A6) constructed from $\mathbf{B}_{N}^{\text {eff }}$ and the corresponding rate equation [Eqs. (A10.1) and (A10.2)] can be established by evaluating the electron spin vector $\langle\hat{\mathbf{S}}\rangle_{t}$ at time $t$. Solving the equation leads to the eigen-state population and nuclear-spin polarization at time $t+\Delta t$. We note the key simplification here is to treat each nuclear spin independently from other nuclear spins but all coupled to the electron spin. The same scenario is also applied in the central spin dynamics in Refs. [12] and [13]. On the other hand, the nuclear-spin dipole-dipole interaction that couples nuclear spins is assumed to cause homogenous relaxation of the nuclear-spin polarization, which is absorbed to $T_{1}$ in Eq. (A10). For electron spin, the iteration is through the Bloch Eq. (A6). Taking into account contributions from the spin polarization of all nuclei at time $t, \mathbf{B}_{e}^{\text {eff }}$ can be calculated, which parameterizes the Bloch equation and determines the steady-state electronspin vector at time $t+\Delta t$. We note that, in this approach, the electron- and nuclear-spin system are treated independently. The feedback is established through the iteration process. Additionally, the phase coherence of the nuclear spin is not preserved in Eqs. (A10.1) and (A10.2). A similar approach with independently treated electron and nuclear spin has been reported before and has yielded satisfactory results [13]. In another aspect, since we are simultaneously dealing with a large number of nuclear spins with different quadrupole axes $\mathbf{n}_{i}$, the phase coherence of individual nuclear spins is expected to be averaged out.

\section{APPENDIX B: ESTIMATION OF SHEAR STRAIN FROM THE ANISOTROPIC EXCHANGE INTERACTION}

The magnitude of shear strain can be estimated from the polarization of the bright neutral exciton states that split by an AEI. One can consider the Bir-Pikus Hamiltonian for strained GaAs in the Hilbert space spanned by the heavy hole (HH) and light hole (LH) basis. Typically, the uniaxial strain $\varepsilon_{u}=\left(\varepsilon_{x x}+\varepsilon_{y y}\right) / 2-\varepsilon_{z z}$ and confinement effect in QD results in a splitting of the HH and LH states that can be as large as $120 \mathrm{meV}$ [44]. The exciton ground state of QDs are mostly contributed by the HH-like spinup state $|\mathrm{HH}, \Uparrow\rangle$ and spin-down state $|\mathrm{HH}, \Downarrow\rangle$ with slight mixing with the LH states in the presence of biaxial strain $\varepsilon_{B}=\varepsilon_{x x}-\varepsilon_{y y}$ and shear strain $\varepsilon_{x z}$ and $\varepsilon_{y z}$,

$$
\begin{aligned}
|\mathrm{HH}, \Uparrow\rangle= & \frac{1}{M}\left[\left|\frac{3}{2},+\frac{3}{2}\right\rangle+\frac{1}{2 Q}\right. \\
& \left.\times\left(R^{*}\left|\frac{3}{2},-\frac{1}{2}\right\rangle-S^{*}\left|\frac{3}{2},+\frac{1}{2}\right\rangle\right)\right], \\
|\mathrm{HH}, \Downarrow\rangle= & \frac{1}{M}\left[\left|\frac{3}{2},-\frac{3}{2}\right\rangle+\frac{1}{2 Q}\right. \\
& \left.\times\left(R\left|\frac{3}{2},+\frac{1}{2}\right\rangle+S\left|\frac{3}{2},-\frac{1}{2}\right\rangle\right)\right] .
\end{aligned}
$$

Here, $M$ is the normalization factor and the strain components enter through the parameters,

$$
\begin{gathered}
Q=b \varepsilon_{u}+\frac{\Delta}{2}, \\
R=\frac{\sqrt{3} b}{2} \varepsilon_{B}-i d \varepsilon_{x y},
\end{gathered}
$$




$$
S=d\left(\varepsilon_{x z}-i \varepsilon_{y z}\right),
$$

where $\Delta$ is the confinement-induced HH-LH splitting, $b=$ $-1.95 \mathrm{eV}$ and $d=-4.51 \mathrm{eV}$ are the deformation potential taken from Ref. [45]. The HH-LH mixing has an impact on the polarization of the neutral exciton states in a single QDS, that can be measured by micro-PL. Taking the basis of $|\mathrm{HH}, \uparrow\rangle \otimes|\mathrm{CB}, \uparrow\rangle,|\mathrm{HH}, \downarrow\rangle \otimes|\mathrm{CB}, \downarrow\rangle,|\mathrm{HH}, \Uparrow\rangle \otimes$ $|\mathrm{CB}, \downarrow\rangle$, and $|\mathrm{HH}, \downarrow\rangle \otimes|\mathrm{CB}, \uparrow\rangle$, where $\mathrm{CB}$ specifies the conduction band electron state, one can obtain the neutral exciton states with the effective exchange Hamiltonian $H_{\mathrm{ex}}=\sum_{i=x, y, z} a_{i} s_{i} j_{i}+b_{i} s_{i} j_{i}^{3}$ [27]. It yields two linearly polarized bright exciton states and two dark states that are separated by the bright-dark state splitting. The emission intensity of the bright exciton states can be simulated as a function of detection polarization angle, which yields the results shown in Fig. 5(a). For this simulation, we take the HH-LH splitting $2 Q=120 \mathrm{meV}$ and vanishing biaxial strain $\varepsilon_{B}=0$. We define a shear-strain component $\varepsilon_{x z}-i \varepsilon_{y z}=\varepsilon_{s} e^{i \gamma}$. In Fig. 5(a), we assume $\varepsilon_{s}=0.02$ and $\gamma=30^{\circ}$. We also assume $\varepsilon_{x y}=0$, since $\varepsilon_{x y}$ only rotates the strain field in the $x-y$ plane, of which the effect can be accommodated by varying $\gamma$. The exchange parameter is chosen so that the bright-dark splitting and AEI is $200 \mu \mathrm{eV}$ and $30 \mu \mathrm{eV}$ without the shear strain [27,41]. The shear strain $\varepsilon_{s} e^{i \gamma}$ breaks the orthogonality of the bright exciton states. In Fig. 5(a), we introduce an angle $\theta_{X^{0}} \leq 90^{\circ}$ between the polarization directions of the exciton states. In Fig. 5(b), we show the simulated $\theta_{X^{0}}$ as a function of $\gamma$, which is plotted for different $\varepsilon_{s}$ values from 0 to 0.024 . It shows that the lower bound of $\theta_{X^{0}}$ correlates with the magnitude of shear strain $\varepsilon_{s}$.

In Fig. 5(a), we show typical polarization angular dependence of the PL intensity measured from the bright exciton states of an individual QDS. The experimental data (the symbols) is fitted by the sine square function (the solid lines) to yield $\theta_{X^{0}}$ for each pair of states. The statistics of the as-extracted $\theta_{X^{0}}$ is plotted in Figs. 5(c)-5(e) for QC, $\mathrm{QR}$, and $\mathrm{DQD}$, respectively. We find that the lower bound of $\theta_{X^{0}}$ in DQD is statistically higher than that in the QC and QR samples, suggesting a more pronounced shear-strain component in the latter.

Comparing Fig. 5(c) with Fig. 5(c) and assuming that the $\gamma$ in QCs is random enough, the low-bound value $\theta_{X^{0}}=67.5^{\circ}$ actually corresponds to a shear-strain strength of $\varepsilon_{s}=0.024$, which is rather large compared with the typical diagonal strain component $\varepsilon_{z z} \approx 0.03$ and $\varepsilon_{x x}=$ $\varepsilon_{y y} \approx-0.03$ reported in the literature [46]. However, we note that this result is not surprising, as the atomic shear strain higher than 0.02 has been seen in single QDs in the literature [46,47]. However, in single QDs, $\epsilon_{s}$ is mostly distributed at the interface between the QD and the host material where the nuclear spins have weak influence on the electron spin. More importantly, owing to typical symmetry of $C_{2 v}$ or higher, the shear-strain components at opposite interfaces have different signs such that its effect on both the NQI and anisotropic exchange interaction is averaged out [48]. The QDS investigated in this work has symmetry of $C_{s}$ or lower such that a net effect of shear strain is preserved. If assuming vanishing $\varepsilon_{x y}$, we can diagonalize the strain tensor and obtain tilting of the principal strain axis by $20^{\circ}$ from the $z$ axis.

[1] R. Dzhioev and V. Korenev, Stabilization of the ElectronNuclear Spin Orientation in Quantum Dots by the Nuclear Quadrupole Interaction, Phys. Rev. Lett. 99, 037401 (2007).

[2] G. Sallen, S. Kunz, T. Amand, L. Bouet, T. Kuroda, T. Mano, D. Paget, O. Krebs, X. Marie, K. Sakoda, and B. Urbaszek, Nuclear magnetization in gallium arsenide quantum dots at zero magnetic field, Nat. Commun. 5, 3268 (2014).

[3] J. R. Petta, J. M. Taylor, A. C. Johnson, A. Yacoby, M. D. Lukin, C. M. Marcus, M. P. Hanson, and A. C. Gossard, Dynamic Nuclear Polarization with Single Electron Spins, Phys. Rev. Lett. 100, 067601 (2008).

[4] A. Bracker, E. Stinaff, D. Gammon, M. Ware, J. Tischler, A. Shabaev, A. Efros, D. Park, D. Gershoni, V. Korenev, and I. Merkulov, Optical Pumping of the Electronic and Nuclear Spin of Single Charge-Tunable Quantum Dots, Phys. Rev. Lett. 94, 047402 (2005).

[5] B. Urbaszek, X. Marie, T. Amand, O. Krebs, P. Voisin, P. Maletinsky, A. Högele, and A. Imamoglu, Nuclear spin physics in quantum dots: An optical investigation, Rev. Mod. Phys. 85, 79 (2013).

[6] D. Awschalom, D. Loss, N. Samarth, Semiconductor Spintronics and Quantum Computation (Springer-Verlag Berlin Heidelberg, Heidelberg, 2002).

[7] J. J. Pla, K. Y. Tan, J. P. Dehollain, W. H. Lim, J. J. L. Morton, F. A. Zwanenburg, D. N. Jamieson, A. S. Dzurak, and A. Morello, High-fidelity readout and control of a nuclear spin qubit in silicon, Nature 496, 334 (2013).

[8] B. Eble, O. Krebs, A. Lemaître, K. Kowalik, A. Kudelski, P. Voisin, B. Urbaszek, X. Marie, and T. Amand, Dynamic nuclear polarization of a single charge-tunable InAs/GaAs quantum dot, Phys. Rev. B 74, 081306 (2006).

[9] F. Hoehne, L. Dreher, D. P. Franke, M. Stutzmann, L. S. Vlasenko, K. M. Itoh, and M. S. Brandt, Submillisecond Hyperpolarization of Nuclear Spins in Silicon, Phys. Rev. Lett. 114, 117602 (2015).

[10] P. Maletinsky, M. Kroner, and A. Imamoglu, Breakdown of the nuclear-spin-temperature approach in quantum-dot demagnetization experiments, Nat. Phys. 5, 407 (2009).

[11] O. Krebs, P. Maletinsky, T. Amand, B. Urbaszek, A. Lemaître, P. Voisin, X. Marie, A. Imamoglu, and A. Lema1, Anomalous Hanle Effect due to Optically Created Transverse Overhauser Field in Single InAs/GaAs Quantum Dots, Phys. Rev. Lett. 104, 056603 (2010).

[12] A. Bechtold, D. Rauch, F. Li, T. Simmet, P.-L. Ardelt, A. Regler, K. Müller, N. A. Sinitsyn, and J. J. Finley, Three-stage decoherence dynamics of an electron spin qubit in an optically active quantum dot, Nat. Phys. 11, 1005 (2015). 
[13] N. A. Sinitsyn, Y. Li, S. A. Crooker, A. Saxena, and D. L. Smith, Role of Nuclear Quadrupole Coupling on Decoherence and Relaxation of Central Spins in Quantum Dots, Phys. Rev. Lett. 109, 166605 (2012).

[14] M. S. Kuznetsova, R. V. Cherbunin, I. Y. Gerlovin, I. V. Ignatiev, S. Y. Verbin, D. R. Yakovlev, D. Reuter, A. D. Wieck, and M. Bayer, Spin dynamics of quadrupole nuclei in InGaAs quantum dots, Phys. Rev. B 95, 155312 (2017).

[15] J. Hackmann, P. Glasenapp, A. Greilich, M. Bayer, and F. B. Anders, Influence of the Nuclear Electric Quadrupolar Interaction on the Coherence Time of Hole and Electron Spins Confined in Semiconductor Quantum Dots, Phys. Rev. Lett. 115, 207401 (2015).

[16] I. M. Griffiths, H. Huang, A. Rastelli, M. S. Skolnick, and E. A. Chekhovich, Complete characterization of GaAs gradient-elastic tensors and reconstruction of internal strain in GaAs/AlGaAs quantum dots using nuclear magnetic resonance, Phys. Rev. B 99, 125304 (2019).

[17] E. A. Chekhovich, K. V. Kavokin, J. Puebla, A. B. Krysa, M. Hopkinson, A. D. Andreev, A. M. Sanchez, R. Beanland, M. S. Skolnick, and A. I. Tartakovskii, Structural analysis of strained quantum dots using nuclear magnetic resonance, Nat. Nanotechnol. 7, 646 (2012).

[18] E. A. Chekhovich, M. Hopkinson, M. S. Skolnick, and A. I. Tartakovskii, Suppression of nuclear spin bath fluctuations in self-assembled quantum dots induced by inhomogeneous strain, Nat. Commun. 6, 6348 (2015).

[19] C. Bulutay, Quadrupolar spectra of nuclear spins in strained $\mathrm{In}_{x} \mathrm{Ga}_{1-x}$ As quantum dots, Phys. Rev. B 85, 115313 (2012).

[20] C. Bulutay, E. A. Chekhovich, and A. I. Tartakovskii, Nuclear magnetic resonance inverse spectra of InGaAs quantum dots: Atomistic level structural information, Phys. Rev. B 90, 205425 (2014).

[21] See Supplemental Material at http://link.aps.org/supplem ental/10.1103/PhysRevApplied.14.044019 for schematic illustration of measurement setup; signature of $\mathrm{C}$ acceptor; PL and $P_{\mathrm{PL}}$ spectra from the QDSs; angular-dependent Hanle curves from the QRs, DQDs, and QCs; low-field magneto-optical features in a longitudinal magnetic field; effect of magnetic field ramping direction; simulation of the effect of biaxial NQI; build up of DNP; simulation of angular-dependent Hanle curves at low-field range; simulation of DNP and Knight field features; magneto-optical results from the SQD sample; computer-generated imbalanced atomic configurations; growth of QDSs; effect of the biaxiality on the oblique NQI.

[22] S. Suraprapapich, S. Panyakeow, and C. W. Tu, Effect of arsenic species on the formation of $(\mathrm{Ga}) \mathrm{InAs}$ nanostructures after partial capping and regrowth, Appl. Phys. Lett. 90, 183112 (2007).

[23] P. Offermans, P. M. Koenraad, J. H. Wolter, D. Granados, J. M. García, V. M. Fomin, V. N. Gladilin, and J. T. Devreese, Atomic-scale structure of self-assembled $\operatorname{In}(\mathrm{Ga})$ As quantum rings in GaAs, Appl. Phys. Lett. 87, 131902 (2005).

[24] X. Zhou, S. Sanwlani, W. Liu, J. H. Lee, Z. M. Wang, G. Salamo, and M. F. Doty, Spectroscopic signatures of manybody interactions and delocalized states in self-assembled lateral quantum dot molecules, Phys. Rev. B 84, 205411 (2011).
[25] M. Ozeki, K. Nakai, K. Dazai, and O. Ryuzan, Photoluminescence study of carbon doped gallium arsenide, Jpn. J. Appl. Phys. 13, 1121 (1974).

[26] F. Meier and B. Zakharchenya, Optical Orientation (North Holland, Amsterdam, 1984).

[27] M. Bayer, G. Ortner, O. Stern, A. Kuther, A. Gorbunov, A. Forchel, P. Hawrylak, S. Fafard, K. Hinzer, T. Reinecke, S. Walck, J. Reithmaier, F. Klopf, and F. Schäfer, Fine structure of neutral and charged excitons in selfassembled $\mathrm{In}(\mathrm{Ga}) \mathrm{As} /(\mathrm{Al}) \mathrm{GaAs}$ quantum dots, Phys. Rev. B 65, 195315 (2002).

[28] R. Dzhioev, V. Korenev, B. Zakharchenya, D. Gammon, A. Bracker, J. Tischler, and D. Katzer, Optical orientation and the Hanle effect of neutral and negatively charged excitons in GaAs $/ \mathrm{Al}_{x} G a_{1-x}$ As quantum wells, Phys. Rev. B 66, 153409 (2002).

[29] J. Beyer, P. H. Wang, I. a Buyanova, S. Suraprapapich, C. W. Tu, and W. M. Chen, Effects of a longitudinal magnetic field on spin injection and detection in InAs/GaAs quantum dot structures, J. Phys. Condens. Matter 24, 145304 (2012).

[30] C. Lai, P. Maletinsky, A. Badolato, and A. Imamoglu, Knight-field-enabled Nuclear Spin Polarization in Single Quantum Dots, Phys. Rev. Lett. 96, 167403 (2006).

[31] S. Y. Verbin, I. Y. Gerlovin, I. V. Ignatiev, M. S. Kuznetsova, R. V. Cherbunin, K. Flisinski, D. R. Yakovlev, and M. Bayer, Dynamics of nuclear polarization in InGaAs quantum dots in a transverse magnetic field, J. Exp. Theor. Phys. 114, 681 (2012).

[32] I. A. Merkulov, A. L. Efros, and M. Rosen, Electron spin relaxation by nuclei in semiconductor quantum dots, Phys. Rev. B 65, 205309 (2002).

[33] M. S. Kuznetsova, K. Flisinski, I. Y. Gerlovin, I. V. Ignatiev, K. V. Kavokin, S. Y. Verbin, D. R. Yakovlev, D. Reuter, A. D. Wieck, and M. Bayer, Hanle effect in (In, Ga)As quantum dots: Role of nuclear spin fluctuations, Phys. Rev. B 87, 235320 (2013).

[34] M. I. D’yakonov, V. I. Perel', V. L. Berkovits, and V. I. Safarov, Optical effects due to polarization of nuclei in semiconductors, Sov. Phys. JETP 40, 950 (1975).

[35] T. Auer, R. Oulton, A. Bauschulte, D. R. Yakovlev, M. Bayer, S. Y. Verbin, R. V. Cherbunin, D. Reuter, and A. D. Wieck, Measurement of the Knight field and local nuclear dipole-dipole field in an InGaAs/GaAs quantum dot ensemble, Phys. Rev. B 80, 205303 (2009).

[36] A. Högele, M. Kroner, S. Seidl, K. Karrai, M. Atatüre, J. Dreiser, A. Imamoğlu, R. J. Warburton, A. Badolato, B. D. Gerardot, and P. M. Petroff, Spin-selective optical absorption of singly charged excitons in a quantum dot, Appl. Phys. Lett. 86, 221905 (2005).

[37] M. I. Dyakonov, I. A. Merkulov, and V. I. Perel, Opticalorientation anisotropy produced in semiconductors by quadrupole splitting of the spin levels of the lattice nuclei, Sov. Phys. JETP 49, 160 (1979).

[38] P. Maletinsky, A. Badolato, and A. Imamoglu, Dynamics of Quantum dot Nuclear Spin Polarization Controlled by a Single Electron, Phys. Rev. Lett. 99, 056804 (2007).

[39] R. V. Cherbunin, S. Y. Verbin, T. Auer, D. R. Yakovlev, D. Reuter, A. D. Wieck, I. Y. Gerlovin, I. V. Ignatiev, D. V. Vishnevsky, and M. Bayer, Dynamics of the nuclear spin polarization by optically oriented electrons in a 
(In, Ga)As/GaAs quantum dot ensemble, Phys. Rev. B 80, 035326 (2009).

[40] S. Yamamoto, R. Matsusaki, R. Kaji, and S. Adachi, Inplane nuclear field formation investigated in single selfassembled quantum dots, Phys. Rev. B 97, 075309 (2018).

[41] S. Fillipov, Y. Puttisong, Y. Huang, I. A. Buyanova, S. Suraprapapich, C. W. Tu, and W. M. Chen, Exciton finestructure splitting in self-assembled lateral InAs/GaAs quantum-dot molecular structures, ACS Nano 9, 5741 (2015).

[42] J. R. Petta, A. C. Johnson, J. M. Taylor, E. a Laird, A. Yacoby, M. D. Lukin, C. M. Marcus, M. P. Hanson, and A. C. Gossard, Coherent manipulation of coupled electron spins in semiconductor quantum dots, Science 309, 2180 (2005).

[43] M. Bayer, P. Hawrylak, K. Hinzer, S. Fafard, M. Korkusinski, Z. Wasilewski, O. Stern, and A. Forchel, Coupling and entangling of quantum states in quantum dot molecules, Science 291, 451 (2001).
[44] G. A. Narvaez, G. Bester, and A. Zunger, Dependence of the electronic structure of self-assembled (In, Ga)As/GaAs quantum dots on height and composition, J. Appl. Phys. 98, 043708 (2005).

[45] A. Çakan, C. Sevik, and C. Bulutay, Strained band edge characteristics from hybrid density functional theory and empirical pseudopotentials: GaAs, GaSb, InAs and InSb, J. Phys. D: Appl. Phys. 49, 085104 (2016).

[46] M. Grundmann, O. Stier, and D. Bimberg, InAs/GaAs pyramidal quantum dots: Strain distribution, optical phonons, and electronic structure, Phys. Rev. B 52, 11969 (1995).

[47] A. D. Andreev, J. R. Downes, D. A. Faux, and E. P. O'Reilly, Strain distributions in quantum dots of arbitrary shape, J. Appl. Phys. 86, 297 (1999).

[48] J. Groenen, C. Priester, and R. Carles, Strain distribution and optical phonons in InAs/InP self-assembled quantum dots, Phys. Rev. B 60, 16013 (1999). 